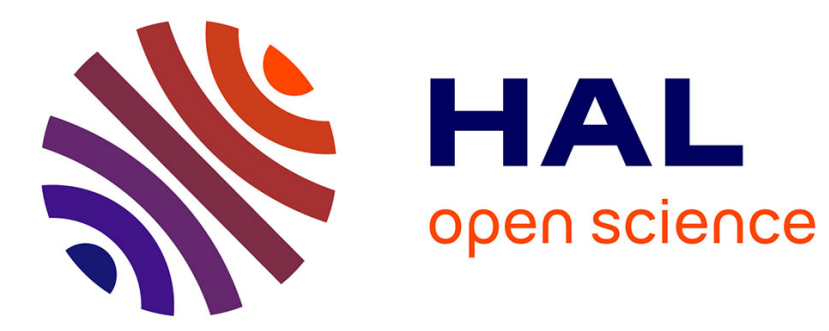

\title{
Good Practice in Large-Scale Learning for Image Classification
}

\author{
Zeynep Akata, Florent Perronnin, Zaid Harchaoui, Cordelia Schmid
}

\section{To cite this version:}

Zeynep Akata, Florent Perronnin, Zaid Harchaoui, Cordelia Schmid. Good Practice in Large-Scale Learning for Image Classification. IEEE Transactions on Pattern Analysis and Machine Intelligence, 2014, 36 (3), pp.507-520. 10.1109/TPAMI.2013.146 . hal-00835810

\section{HAL Id: hal-00835810 https://hal.inria.fr/hal-00835810}

Submitted on 19 Jun 2013

HAL is a multi-disciplinary open access archive for the deposit and dissemination of scientific research documents, whether they are published or not. The documents may come from teaching and research institutions in France or abroad, or from public or private research centers.
L'archive ouverte pluridisciplinaire HAL, est destinée au dépôt et à la diffusion de documents scientifiques de niveau recherche, publiés ou non, émanant des établissements d'enseignement et de recherche français ou étrangers, des laboratoires publics ou privés. 


\title{
Good Practice in Large-Scale Learning for Image Classification
}

\author{
Zeynep Akata, Student Member, IEEE, Florent Perronnin, Zaid Harchaoui, Member, IEEE, \\ and Cordelia Schmid, Fellow, IEEE
}

\begin{abstract}
We benchmark several SVM objective functions for large-scale image classification. We consider one-vs-rest, multi-class, ranking, and weighted approximate ranking SVMs. A comparison of online and batch methods for optimizing the objectives shows that online methods perform as well as batch methods in terms of classification accuracy, but with a significant gain in training speed. Using stochastic gradient descent, we can scale the training to millions of images and thousands of classes. Our experimental evaluation shows that ranking-based algorithms do not outperform the one-vs-rest strategy when a large number of training examples are used. Furthermore, the gap in accuracy between the different algorithms shrinks as the dimension of the features increases. We also show that learning through cross-validation the optimal rebalancing of positive and negative examples can result in a significant improvement for the one-vs-rest strategy. Finally, early stopping can be used as an effective regularization strategy when training with online algorithms. Following these "good practices", we were able to improve the state-of-the-art on a large subset of $10 \mathrm{~K}$ classes and $9 \mathrm{M}$ images of ImageNet from $16.7 \%$ Top-1 accuracy to $19.1 \%$.
\end{abstract}

Index Terms—Large Scale, Fine-Grained Visual Categorization, Image Classification, Ranking, SVM, Stochastic Learning

\section{INTRODUCTION}

Image classification is the problem of assigning one or multiple labels to an image based on its content. This is a standard supervised learning problem: given a training set of labeled images, the goal is to learn classifiers to predict labels of new images. Large-scale image classification has recently received significant interest from the computer vision and machine learning communities [4], [20], [22], [48], [62], [66], [72], [82], [89]. This goes hand-in-hand with large-scale datasets being available. For instance, ImageNet (www.image-net.org) consists of more than $14 \mathrm{M}$ images labeled with almost $22 \mathrm{~K}$ concepts [21], the Tiny images dataset consists of $80 \mathrm{M}$ images corresponding to $75 \mathrm{~K}$ concepts [72] and Flickr contains thousands of groups (www.flickr.com/groups) with thousands (and sometimes hundreds of thousands) of pictures, that can be exploited to learn object classifiers [80], [58].

Standard large-scale image classification pipelines use highdimensional image descriptors in combination with linear classifiers [48], [66]. The use of linear classifiers is motivated by their computational efficiency - a requirement when dealing with a large number of classes and images. High-dimensional descriptors allow separating the data with a linear classifier, i.e., they perform the feature mapping explicitly and avoid using nonlinear kernels. One of the simplest strategies to learn classifiers in the multi-class setting is to train onevs-rest binary classifiers independently for each class. Most image classification approaches have adopted this strategy not

- Z.Akata and F.Perronnin are with the Xerox Research Centre Europe, Meylan, France

- Z.Akata, Z. Harchaoui and C. Schmid are with INRIA Rhone-Alpes, Montbonnot, France. only because of its simplicity, but also because it can be easily parallelized on multiple cores or machines. The two top systems at the ImageNet Large-Scale Visual Recognition Challenge (ILSVRC) 2010 [6] used such an approach [48], [66].

Another approach is to view image classification as a ranking problem: given an image, the goal is to rank the labels according to their relevance. Performance measures such as the top-k accuracy reflect this goal and are used to report results on standard benchmarks, such as ILSVRC. While the one-vsrest strategy is computationally efficient and yields competitive results in practice, it is - at least in theory - clearly suboptimal with respect to a strategy directly optimizing a ranking loss [9], [17], [38], [75], [82], [86], [88].

In this paper, we examine whether these ranking approaches scale well to large datasets and if they improve the performance. We compare the one-vs-rest binary SVM, the multiclass SVM of Crammer and Singer [17] that optimizes top-1 accuracy, the ranking SVM of Joachims [38] that optimizes the rank as well as the recent weighted approximate ranking of Weston et al. [82] that optimizes the top of the ranking list. The datasets we consider are large-scale in the number of classes (up to $10 \mathrm{~K}$ ), images (up to $9 \mathrm{M}$ ) and feature dimensions (up to $130 \mathrm{~K})$. For efficiency reasons we train our linear classifiers using Stochastic Gradient Descent (SGD) algorithms [44] with the primal formulation of the objective functions as in [11], [67] for binary SVMs or in [54] for structured SVMs. By using the exact same optimization framework, we truly focus on the merits of the different objective functions, not on the merits of the particular optimization techniques.

Our experimental evaluation confirms that SGD-based learning algorithms can work as well as batch techniques at a fraction of their cost. It also shows that ranking objective functions seldom outperform the one-vs-rest strategy. Only when a small 


\section{RECOMMENDATIONS FOR LARGE-SCALE IMAGE CLASSIFICATION}

1) Stochastic training: learning with stochastic gradient descent is well-suited for large-scale datasets

2) Class imbalance: optimizing the imbalance parameter in one-vs-rest strategy is a must for competitive performance

3) Early stopping: regularizing through early stopping results in fast training and good generalization performance
4) Step-size: a small-enough step-size w.r.t learning rate is often sufficient for state-of-the-art performance

5) One-vs-rest: one-vs-rest strategy is a flexible option for large-scale image classification

6) Capacity saturation: for a sufficiently large feature representation all strategies lead to similar performance amount of training data is available, did we observe a smallbut-consistent improvement with ranking based methods. The gap between the accuracy of different learning algorithms reduces in case of high-dimensional data. We also experimentally show that for the one-vs-rest strategy carefully tuning the optimal degree of imbalance between the positive and the negative examples can have a significant impact on accuracy. This is observed especially when the feature dimensionality is "small" with respect to the problem complexity, in particular with respect to the number of classes. Furthermore, early stopping can be used as an effective regularization strategy for fast training with SGD. Following these "good practices", we were able to improve the state-of-the-art on a large subset of $10 \mathrm{~K}$ classes and $9 \mathrm{M}$ of images of ImageNet [21] from $16.7 \%$ accuracy to $19.1 \%$. We summarize our findings in the "recommendation box" at the top of this page.

The paper is organized as follows. Section 2 reviews related work in computer vision and machine learning. Section 3 presents the different objective functions for linear classification that we used in our evaluation and Section 4 describes the SGD-based optimization framework. Experimental results are presented in Section 5 for five state-of-the-art datasets: three fine-grained subsets of ImageNet (i.e. Fungus, Ungulate and Vehicles) as well as the ILSVRC 2010 and the very large-scale ImageNet10K datasets. The code used for our experimental evaluation is available at http://lear.inrialpes.fr/software.

A preliminary version of this article has appeared in [55]. This version adds (1) a significantly expanded related work section; (2) experiments for fine-grained classification; (3) an experimental comparison with batch solvers; (4) experiments with few training samples showing the gain of ranking over other objective functions in this setting; (5) experiments on both synthetic data and real data to explore the notion of dataset density; and (6) a comparison with very recent work on deep learning [40], [42].

\section{Related Work}

Recently, many large-scale datasets were collected and made publicly available, such as LabelMe [64], Tiny-images [72] and ImageNet [21]. Most of today's approaches for image classification first extract visual image descriptors and then apply a classifier. In the following, we first review previous work related to visual descriptors, and then give an overview of classification approaches.

\subsection{Visual Descriptors}

A large part of the literature on image classification has been devoted to the design of suitable image representations. A very successful representation paradigm has been to extract local patch-level descriptors from an image, such as SIFT [50] or color descriptors [13], and to aggregate them using operations such as average-pooling or max-pooling. The Bag-of-VisualWords (BOV), which describes an image as a histogram of quantized local descriptors [69], [18], has been the most popular image representation for image classification. It has been improved in many ways over the years. For instance, to go beyond the orderless assumption, Lazebnik et al. [41] proposed Spatial Pyramid Matching (SPM), where statistics over spatial regions are aggregated. Several works have also tried to to go beyond the hard quantization of local descriptors. One possible way to do so is to soft-assign the local descriptors using a generative model of the descriptors [27], [76]. Another approach is based on sparse coding, which forces a descriptor to be assigned to a small number of visual words [87], [12]. While sparse-coding has been shown to yield excellent results, especially in combination with max-pooling [12], it involves a costly iterative optimization process. Hence, efficient alternatives have been sought such as Locality-constrained Linear Coding (LLC) [81], [48] which performs a local embedding of the descriptors. The BOV histogram was shown to be particularly successful in image classification tasks in combination with nonlinear kernel classifiers such as the Support Vector Machine (SVM) (see for instance the results of the successive PASCAL VOC competitions [25]).

However, one of the limitations of nonlinear SVM classifiers is that they do not scale well with the number of training samples. Therefore, several works have proposed to perform an explicit embedding of the image representations in a new space where the BOV histograms are more linearly separable. Maji and Berg [51] proposed mappings for the Intersection Kernel (IK). Vedaldi and Zisserman [77] and Perronnin et al. [57] subsequently generalized this principle to additive classifiers. Gong and Lazebnik [31] benchmarked several feature mapping techniques and showed that data-dependent mappings have an edge over data-independent ones in large-scale scenarios.

While the BOV computes for each local descriptor only simple statistics such as visual word occurrence counts, other approaches have proposed computing higher-order statistics. This includes the Fisher Vector (FV) of Perronnin and Dance [56] which consists in computing the deviation of a set of local descriptors from an average Gaussian Mixture Model (GMM). Perronnin et al. later refined this representation with a set of 
normalization steps [57]. Other higher-order representations have been proposed including the Vector of Locally Aggregated Descriptors (VLAD) [36] and the Super Vector (SV) [90]. Since these techniques perform an explicit embedding of local descriptors in a very high-dimensional space, they typically work well with simple linear classifiers. Chatfield $e t$ al. [16] benchmarked five local descriptor encoding techniques (BOV with hard coding, soft coding and sparse coding as well as SV and FV coding); the FV of [57] was shown to yield the best results on two standard datasets. A weakness of highdimensional representations such as the FV is that they take a lot of space in RAM or on disk [66], [49]. Consequently, they are difficult to scale to large datasets and several works proposed more compact features for classification. One line of work consists in describing an image with a set of highlevel concepts based on object classifiers [80], [73], [7] or object detectors [47]. Coding techniques such as Product Quantization (PQ) [35] have also been used to compress the data [66], [60], [78]. While Sánchez and Perronnin proposed to learn classifiers using Stochastic Gradient Descent (SGD) (see section 2.3) and on-the-fly decompression of training samples [66], Vedaldi and Zisserman showed that classifiers can be learned in the compressed space directly [78].

The standard feature extraction pipeline that aggregates patch descriptors can be criticized as being too shallow to learn complex invariances and high-level concepts. Recently, several large-scale works have considered the learning of features directly from pixel values using deeper architectures [5], [42], [40]. One of the crucial factors to obtain good results when learning deep architectures with millions if not billions of parameters seems to be the availability of vast amounts of training data (see section 10.1 in [5]). In [42], [19], the features are learned using a deep autoencoder which is constructed by replicating three times the same three layers - made of local filtering, local pooling and contrast normalization - thus resulting in an architecture with 9 layers. The learned features were shown to give excellent results in combination with a simple linear classifier. In [40], a deep network with 8 layers was proposed where the first 5 layers are convolutional [43], [45], [34], the remaining three are fully connected and the output of the last fully connected layer is fed to a softmax which produces a distribution over the class labels.

In this work, we will use mostly the FV representation as it was shown to yield excellent results in large-scale classification while requiring only modest computational resources [66]. However, we believe our conclusions to be generic and applicable to other image features.

\subsection{Classifier Strategies}

We underline that in most previous works tackling large-scale visual data sets, the objective function that is optimized is always the same: one binary SVM is learned per class in a one-vs-rest fashion [20], [66], [48], [62]. Some approaches use simple classifiers such as nearest-neighbor (NN) [72]. While exact $\mathrm{NN}$ can provide a competitive accuracy when compared to SVMs as in [82], [20], it is not straightforward to scale to large data sets. On the other hand, Approximate Nearest
Neighbor (ANN) can perform poorly on high-dimensional image descriptors (significantly worse than one-vs-rest SVMs) while still being much more computationally intensive [82]. One-vs-rest (OVR) strategies offer several advantages such as the speed and simplicity as compared to multiclass classifiers (see [61] from Rifkin and Klautau as a defense of OVR). Indeed, OVR classifiers can be trained efficiently by decomposing the problem into independent per class problems.

On the other hand, several versions of multi-class SVMs were proposed. In [85], Weston and Watkins propose a multiclass SVM with a loss function that consists in summing the losses incurred by each class-wise score. Note that this version of multi-class SVM is statistically not consistent for large samples [71]. Lee et al. [46] introduced a statistically consistent version of multi-class SVM, which can be thought of a "hinge-loss" counterpart of multinomial classification. In this work, we use the multiclass SVM formulation of Crammer and Singer from [17], a computationally attractive variant that was proven to be consistent for large-scale problems.

Many alternative approaches for multi-class classification were proposed in the literature. In [24], Dietterich and Bakiri introduce error correcting codes as a basis of multiclass classification. In [1] Allwein et al. combine several multiclass classifiers using AdaBoost. In [79], Vural and Dy approach the problem from the decision trees perspective by partitioning the space in $N-1$ regions.

When the target loss function is not classification accuracy but a more sophisticated performance measure such as meanaverage-precision, a natural approach is to build ranking algorithms. In [38], Joachims proposed a ranking SVM, allowed to rank highly related documents on the higher ranks of the list. In [32], Grangier et al. improve the baseline ranking SVM by giving weights to classifiers. Usunier et al. [75] penalize the loss encountered at the top of the list more than the bottom. Another ranking framework [83] from Weston et al. uses linear classifiers trained with SGD and a novel sampling trick to approximate ranks. To break the time-complexity of training to sub-linear in the number of classes, various approaches have been proposed which employ tree structures [52], [4], [29], [14], [53], [30]. In [8], Beygelzimer et al. create a model for error-limiting reduction in learning. According to this model, the tree (multiclass) reduction has a slightly larger loss rate than binary classifiers. The reader may refer to [63] for an extended survey of top-down approaches for classification.

\subsection{Algorithms and Solvers}

Here, we review the currently publicly available solvers for SVM-like classification and ranking algorithms. We shall not review other approaches, as we do not include them in our benchmarks. There are two main families of algorithms for optimization SVM objectives: batch algorithms, and online/incremental algorithms.

State-of-the-art batch optimization algorithms for non-linear SVMs are based on a variant of coordinate-descent called sequential minimal optimization (SMO)[59]. The main strength of batch approaches is the high robustness to the settings of the algorithms parameters, that is initialization, line-search, 
number of iterations, etc. Such algorithms are implemented in the most popular toolboxes LibSVM [15] from Chang et al., SVM ${ }^{\text {light }}$ [37], and Shogun [28]. State-of-the art batch algorithms for linear SVMs are based on coordinate-descent approaches with second-order acceleration. The widely used LibLinear toolbox [33] from Hsieh et al. provides an efficient implementation of such algorithms. The weakness of batch methods is the difficulty to scale up to large datasets.

State-of-the-art stochastic (online) optimization algorithms for linear SVMs are based on stochastic gradient descent (SGD). The well known online SVM solver Pegasos [67] from Shalew-Shwartz et al. and libSGD [10] from Bottou provide an implementation of these algorithms. The main strength of SGD algorithms is their built-in ability to scale up to large datasets. However, without a careful setting of the parameters, SGD algorithms can have slow convergence and struggle to match the performance of their batch algorithm counterparts. In this paper, we highlight the "golden rules" while using SGD to get competitive performance with batch solvers, and provide empirical evidence of the success of our methodology.

\section{OBJective Functions}

Let $S=\left\{\left(\mathbf{x}_{i}, y_{i}\right), i=1 \ldots N\right\}$ be the training set where $\mathbf{x}_{i} \in \mathcal{X}$ is an image descriptor, $y_{i} \in \mathcal{Y}$ is the associated label and $\mathcal{Y}$ is the set of possible labels. Let $C=|\mathcal{Y}|$ denote the number of classes. We shall always take $\mathcal{X}=\mathbb{R}^{D}$.

Supervised learning corresponds to minimizing the empirical risk with a regularization penalty

$$
\underset{\mathbf{W}}{\operatorname{Minimize}} \frac{\lambda}{2} \Omega(\mathbf{W})+L(S ; \mathbf{W}),
$$

where $\mathbf{W}$ is the weight matrix stacking the weight vectors corresponding to each subproblem. The objective decomposes into the empirical risk

$$
L(S ; \mathbf{W}):=\frac{1}{N} \sum_{i=1}^{N} L\left(\mathbf{x}_{i}, y_{i} ; \mathbf{W}\right)
$$

with $L\left(\mathbf{x}_{i}, y_{i} ; \mathbf{W}\right)$ a surrogate loss of the labeled example $\left(x_{i}, y_{i}\right)$, and the regularization penalty

$$
\Omega(\mathbf{W}):=\sum_{c=1}^{C}\left\|\mathbf{w}_{c}\right\|^{2}
$$

The parameter $\lambda \geq 0$ controls the trade-off between the empirical risk and the regularization. We first briefly review the classical binary SVM. We then proceed with the multiclass, ranking and weighted approximate ranking SVMs. We finally discuss the issue of data re-weighting.

\subsection{Binary One-Vs-Rest SVM (OVR)}

In the case of the one-vs-rest SVM, we assume that we have only two classes and $\mathcal{Y}=\{-1,+1\}$. Let $\mathbb{1}(u)=1$ if $\mathrm{u}$ is true and 0 otherwise. The $0-1$ loss $\mathbb{1}\left(y_{i} \mathbf{w}^{T} x_{i}<0\right)$ is upperbounded by

$$
L_{\mathrm{OVR}}\left(\mathbf{x}_{i}, y_{i} ; \mathbf{w}\right)=\max \left\{0,1-y_{i} \mathbf{w}^{T} \mathbf{x}_{i}\right\}
$$

If we have more than two classes, then one transforms the $C$-class problem into $C$ binary problems and trains independently $C$ one-vs-rest classifiers.

\subsection{Beyond Binary Classification}

In the following sections, we treat the classes jointly and the label set contains more than two labels i.e. $\mathcal{Y}=\{1, \ldots, C\}$. Let $\left\{\mathbf{w}_{c}, c=1 \ldots C\right\}$ denote the $C$ classifiers corresponding to each of the $C$ classes. In this case, $\mathbf{W}$ is a $C \times D$ dimensional vector obtained by concatenating the different $w_{c}$ 's. We denote by $\Delta(y, \bar{y})$ the loss incurred for assigning label $\bar{y}$ while the correct label was $y$. In this work, we focus on the $0 / 1$ loss, i.e. $\Delta(y, \bar{y})=0$ if $y=\bar{y}$ and 1 otherwise. In what follows, we assume that we have one label per image to simplify the representation.

Multiclass SVM (MUL). There exist several flavors of the multiclass SVM such as the Weston and Watkins [84] and the Crammer and Singer [17] formulations (see [71] for a comprehensive review). Both variants propose a convex surrogate loss to $\Delta\left(y_{i}, \hat{y}_{i}\right)$ with

$$
\hat{y}_{i}=\arg \max _{y} \mathbf{w}_{y}^{T} x_{i},
$$

i.e. the loss incurred by taking the highest score as the predicted label. We choose the Crammer and Singer formulation, corresponding to

$$
L_{\mathrm{MUL}}\left(\mathbf{x}_{i}, y_{i} ; \mathbf{w}\right)=\max _{y}\left\{\Delta\left(y_{i}, y\right)+\mathbf{w}_{y}^{T} x_{i}\right\}-\mathbf{w}_{y_{i}}^{T} x_{i}
$$

which provides a tight bound on the misclassification error [74]. Note that this can be viewed as a particular case of the structured SVM [74].

Ranking SVM (RNK). Joachims [38] considers the problem of ordering pairs of documents. Adapting the ranking framework to our problem the goal is, given a sample $\left(\mathbf{x}_{i}, y_{i}\right)$ and a label $y \neq y_{i}$, to enforce $\mathbf{w}_{y_{i}} \mathbf{x}_{i}>\mathbf{w}_{y}^{T} \mathbf{x}_{i}$. The rank of label $y$ for sample $\mathbf{x}$ can be written as:

$$
r(\mathbf{x}, y)=\sum_{c=1}^{C} \mathbb{1}\left(\mathbf{w}_{c}^{T} \mathbf{x} \geq \mathbf{w}_{y}^{T} \mathbf{x}\right)
$$

Given the triplet $\left(\mathbf{x}_{i}, y_{i}, y\right), \mathbb{1}\left(\mathbf{w}_{c}^{T} \mathbf{x} \geq \mathbf{w}_{y}^{T} \mathbf{x}\right)$ is upperbounded by:

$$
L_{\text {tri }}\left(\mathbf{x}_{i}, y_{i}, y ; \mathbf{w}\right)=\max \left\{0, \Delta\left(y_{i}, y\right)-\mathbf{w}_{y_{i}}^{T} \mathbf{x}_{i}+\mathbf{w}_{y}^{T} \mathbf{x}_{i}\right\}
$$

Therefore, the overall loss of $\left(\mathbf{x}_{i}, y_{i}\right)$ writes as:

$$
L_{\mathrm{RNK}}\left(\mathbf{x}_{i}, y_{i} ; \mathbf{w}\right)=\sum_{y=1}^{C} \max \left\{0, \Delta\left(y_{i}, y\right)-\left(\mathbf{w}_{y_{i}}-\mathbf{w}_{y}\right)^{T} \mathbf{x}_{i}\right\}
$$

Weighted Approximate Ranking SVM (WAR). An issue with the Ranking SVM (RNK) is that the loss is the same when going from rank 99 to 100 or from rank 1 to rank 2 . However, in most practical applications, one is interested in the top of the ranked list. As an example, in ILSVRC the measure used during the competition is a loss at rank 5. Usunier et al. [75] therefore proposed to minimize a function of the rank which gives more weight to the top of the list.

Let $\alpha_{1} \geq \alpha_{2} \geq \ldots \alpha_{C} \geq 0$ be a set of $C$ coefficients. For sample $\left(\mathbf{x}_{i}, y_{i}\right)$ the loss is $\ell_{r\left(\mathbf{x}_{i}, y_{i}\right)}$ with $\ell$ defined as

$$
\ell_{k}=\sum_{j=1}^{k} \alpha_{j}
$$


The penalty incurred by going from rank $k$ to $k+1$ is $\alpha_{k}$. Hence, a decreasing sequence $\left\{\alpha_{j}\right\}_{j \geq 1}$ implies that a mistake on the rank when the true rank is at the top of the list incurs a higher loss than a mistake on the rank when the true rank is lower in the list. We note that this Ordered Weighted Ranking (OWR) objective function is generic and admits as special cases the multiclass SVM of Crammer and Singer $\left(\alpha_{1}=1\right.$ and $\alpha_{j}=0$ for $j \geq 2$ ) and the ordered pairwise ranking SVM of Joachims $\left(\alpha_{j}=1, \forall i\right)$.

While [75] proposes an upper-bound on the loss, Weston et al. [82] propose an approximation. We follow [82] which is more amenable to large-scale optimization and write:

$$
L_{\mathrm{WAR}}\left(\mathbf{x}_{i}, y_{i} ; \mathbf{w}\right)=\sum_{y=1}^{C} \ell_{r_{\Delta}\left(\mathbf{x}_{i}, y_{i}\right)} \frac{L_{\mathrm{tri}}\left(\mathbf{x}_{i}, y_{i}, y ; \mathbf{w}\right)}{r_{\Delta}\left(\mathbf{x}_{i}, y_{i}\right)},
$$

where

$$
r_{\Delta}(\mathbf{x}, y)=\sum_{c=1}^{C} \mathbb{1}\left(\mathbf{w}_{c}^{T} x+\Delta(y, c) \geq \mathbf{w}_{y}^{T} x\right)
$$

is a regularized rank. Following [82], we choose $\alpha_{j}=1 / j$. As opposed to other works [86], [88], this does not optimize directly standard information retrieval measures such as Average Precision (AP). However, it mimics their behavior by putting emphasis on the top of the list, works well in practice and is highly scalable.

Data Rebalancing. When the training set is unbalanced, i.e. when some classes are significantly more populated than others, it can be beneficial to reweight the data. This imbalance can be extreme in the one-vs-rest case when one has to deal with a large number of classes $C$ as the imbalance between the positive class and the negative class is on average $C-1$. In the binary case, the reweighting can be performed by introducing a parameter $\rho$ and the empirical risk then writes as:

$$
\frac{\rho}{N_{+}} \sum_{i \in I_{+}} L_{\mathrm{OVR}}\left(\mathbf{x}_{i}, y_{i} ; \mathbf{w}\right)+\frac{1-\rho}{N_{-}} \sum_{i \in I_{-}} L_{\mathrm{OVR}}\left(\mathbf{x}_{i}, y_{i} ; \mathbf{w}\right)
$$

where $I_{+}$(resp. $I_{-}$) is the set of indices of the positive (resp. negative) samples and $N_{+}$(resp. $N_{-}$) is the cardinality of this set. Note that $\rho=1 / 2$ corresponds to the natural rebalancing of the data, i.e. in such a case one gives as much weight to positives and negatives.

When training all classes simultaneously, introducing one parameter per class is computationally intractable. It would require cross-validating $C$ parameters jointly, including the regularization parameter. In such a case, the natural rebalancing appears to be the most natural choice. In the multiclass case, the empirical loss becomes:

$$
\frac{1}{C} \sum_{c=1}^{C} \frac{1}{N_{c}} \sum_{i \in I_{c}} L_{\mathrm{MUL}}\left(\mathbf{x}_{i}, y_{i} ; \mathbf{w}\right)
$$

where $I_{c}=\left\{i: y_{i}=c\right\}$ and $N_{c}$ is the cardinality of this set. One can perform a similar rebalancing in the case of $L_{\mathrm{RNK}}$ and $L_{\mathrm{WAR}}$.

\section{Optimization Algorithms}

We employ Stochastic Gradient Descent (SGD) to learn linear classifiers in the primal [67], [11]. Such an approach has recently gained popularity in the computer vision community for large-scale learning [57], [58], [82], [66], [48], [62]. In the following, we describe the optimization algorithms for various objective functions and give implementation details.

\subsection{Stochastic Training}

Training with Stochastic Gradient Descent (SGD) consists at each step in choosing a sample at random and updating the parameters $\mathbf{w}$ using a sample-wise estimate of the regularized risk. In the case of $R_{\mathrm{OVR}}$ and $R_{\mathrm{MUL}}$, the sample is simply a pair $\left(\mathbf{x}_{i}, y_{i}\right)$ while in the case of $R_{\mathrm{RNK}}$ and $R_{\mathrm{WAR}}$ it consists of a triplet $\left(\mathbf{x}_{i}, y_{i}, \bar{y}\right)$ where $\bar{y} \neq y_{i}$. Let $z_{t}$ denote the sample drawn at step $t$ (whether it is a pair or a triplet) and let $R\left(z_{t} ; \mathbf{w}\right)$ be the sample-wise estimate of the regularized risk. $\mathbf{w}$ is updated as follows:

$$
\mathbf{w}^{(t)}=\mathbf{w}^{(t-1)}-\eta_{t} \nabla_{\mathbf{w}=\mathbf{w}^{(t-1)}} R\left(z_{t} ; \mathbf{w}\right)
$$

where $\eta_{t}$ is the step size.

We provide in Table 1 the sampling and update procedures for the objective functions used in our evaluation, assuming no rebalancing of the data. For $R_{\mathrm{OVR}}, R_{\mathrm{MUL}}$ and $R_{\mathrm{RNK}}$, these equations are straightforward and optimize exactly the regularized risk. For $R_{\mathrm{WAR}}$ it is only approximate as it does not compute exactly the value of $r_{\Delta}\left(\mathbf{x}_{i}, y_{i}\right)$, but estimates it from the number of samples $k$ which were drawn before a violating sample $\bar{y}$ such that $L_{\text {tri }}\left(x_{i}, y_{i}, \bar{y} ; \mathbf{w}\right)>0$ was found. If $k$ samples were drawn, then:

$$
r_{\Delta}\left(\mathbf{x}_{i}, y_{i}\right) \approx\left\lfloor\frac{C-1}{k}\right\rfloor .
$$

For a large number of classes, this approximate procedure is significantly faster than the exact one (see [82] for more details). Note also that we implement the regularization by penalizing the squared norm of $\mathbf{w}$, while [82] actually bounds the norm of $\mathbf{w}$. We tried both strategies and observed that they provide similar results in practice.

\subsection{Implementation Details}

We use as basis for our code the SGD library for binary classification available on Bottou's website (version 1.3)[10]. This is an optimized code that includes fast linear algebra and a number of optimizations such as the use of a scale variable to update $\mathbf{w}$ only when a loss is incurred.

We now discuss a number of implementation details.

Bias. Until now, we have not considered the bias in our objective functions. This corresponds to an additional parameter per class. Following common practice, we add one constant feature to each observation $\mathbf{x}_{i}$. As is the case in Bottou's code, we do not regularize this additional dimension.

Stopping Criterion. Since at each step in SGD we have a noisy estimate of the objective function, this value cannot be used for stopping. Therefore, in all our experiments, we use a validation set and stop iterating when the accuracy does not increase by more than a threshold $\theta$. 


\begin{tabular}{|c|c|c|}
\hline & Sampling & Update \\
\hline$\overline{R_{\mathrm{OVR}}}$ & Draw $\left(\mathbf{x}_{i}, y_{i}\right)$ from $S$. & $\begin{array}{l}\delta_{i}=1 \text { if } L_{\mathrm{OVR}}\left(\mathbf{x}_{i}, y_{i} ; \mathbf{w}\right)>0,0 \text { otherwise. } \\
\mathbf{w}^{(t)}=\left(1-\eta_{t} \lambda\right) \mathbf{w}^{(t-1)}+\eta_{t} \delta_{i} \mathbf{x}_{i} y_{i}\end{array}$ \\
\hline$R_{\mathrm{MUL}}$ & Draw $\left(\mathbf{x}_{i}, y_{i}\right)$ from $S$ & $\begin{array}{l}\bar{y}=\arg \max _{y} \Delta\left(y_{i}, y\right)+\mathbf{w}_{y}^{\prime} \mathbf{x}_{i} \text { and } \delta_{i}= \begin{cases}1 & \text { if } \bar{y} \neq y_{i} \\
0 & \text { otherwise. }\end{cases} \\
\mathbf{w}_{y}^{(t)}=\left\{\begin{aligned} \mathbf{w}_{y}^{(t-1)}\left(1-\eta_{t} \lambda\right)+\delta_{i} \eta_{t} \mathbf{x}_{i} & \text { if } y=y_{i} \\
\mathbf{w}_{y}^{(t-1)}\left(1-\eta_{t} \lambda\right)-\delta_{i} \eta_{t} \mathbf{x}_{i} & \text { if } y=\bar{y} \\
\mathbf{w}_{y}^{(t-1)}\left(1-\eta_{t} \lambda\right) & \text { otherwise. }\end{aligned}\right.\end{array}$ \\
\hline$\overline{R_{\mathrm{RNK}}}$ & $\begin{array}{l}\text { Draw }\left(\mathbf{x}_{i}, y_{i}\right) \text { from } S \\
\text { Draw } \bar{y} \neq y_{i} \text { from } \mathcal{Y} .\end{array}$ & $\begin{array}{l}\delta_{i}=1 \text { if } L_{\text {tri }}\left(\mathbf{x}_{i}, y_{i}, \bar{y} ; \mathbf{w}\right)>0,0 \text { otherwise. } \\
\mathbf{w}_{y}^{(t)}=\left\{\begin{aligned} \mathbf{w}_{y}^{(t-1)}\left(1-\eta_{t} \lambda\right)+\delta_{i} \eta_{t} \mathbf{x}_{i} & \text { if } y=y_{i} \\
\mathbf{w}_{y}^{(t-1)}\left(1-\eta_{t} \lambda\right)-\delta_{i} \eta_{t} \mathbf{x}_{i} & \text { if } y=\bar{y} \\
\mathbf{w}_{y}^{(t-1)}\left(1-\eta_{t} \lambda\right) & \text { otherwise. }\end{aligned}\right.\end{array}$ \\
\hline$R_{\text {WAR }}$ & $\begin{array}{l}\text { Draw }\left(\mathbf{x}_{i}, y_{i}\right) \text { from } S . \\
\text { For } k=1,2, \ldots, C-1 \text {, do: } \\
\left\{\begin{array}{l}\text { Draw } \bar{y} \neq y_{i} \text { from } \mathcal{Y} . \\
\text { If } L_{\text {tri }}\left(\mathbf{x}_{i}, y_{i}, \bar{y} ; \mathbf{w}\right)>0 \text {, break. }\end{array}\right.\end{array}$ & $\begin{array}{l}\delta_{i}=1 \text { if } \bar{y} \text { s.t. } L_{\text {tri }}\left(\mathbf{x}_{i}, y_{i}, \bar{y} ; \mathbf{w}\right)>0 \text { was sampled, } 0 \text { otherwise. } \\
\mathbf{w}_{y}^{(t)}=\left\{\begin{aligned} \mathbf{w}_{y}^{(t-1)}\left(1-\eta_{t} \lambda\right)+\delta_{i} \ell_{\left\lfloor\frac{C-1}{k}\right\rfloor} \eta_{t} \mathbf{x}_{i} & \text { if } y=y_{i} \\
\mathbf{w}_{y}^{(t-1)}\left(1-\eta_{t} \lambda\right)-\delta_{i} \ell_{\left\lfloor\left\lfloor\frac{C-1}{k}\right\rfloor\right.} \eta_{t} \mathbf{x}_{i} & \text { if } y=\bar{y} \\
\mathbf{w}_{y}^{(t-1)}\left(1-\eta_{t} \lambda\right) & \text { otherwise. }\end{aligned}\right.\end{array}$ \\
\hline
\end{tabular}

TABLE 1

Sampling and update equations for various objective functions.

Regularization. While a vast majority of works on largemargin classification regularize explicitly by penalizing the squared norm of $w$ or by bounding it, regularizing implicitly by early stopping is another option [2]. In such a case, one sets $\lambda=0$ and iterates until the performance converges (or starts descreasing) on a validation set. In our experiments, applying this strategy yields competitive results.

Step Size. To guarantee converge to the optimum, the sequence of step sizes should satisfy $\sum_{t=1}^{\infty} \eta_{t}=\infty$ and $\sum_{t=1}^{\infty} \eta_{t}^{2}<\infty$. Assuming $\lambda>0$, the usual choice is $\eta_{t}=1 / \lambda\left(t+t_{0}\right)$, where $t_{0}$ is a parameter. Bottou provides in his code a heuristic to set $t_{0}$. We tried to cross-validate $t_{0}$ but never observed significant improvements. However, we also experimented with a fixed step size $\eta$ as in [2], [82].

Rebalancing. All sampling/update equations in Table 1 are based on non-rebalanced objective functions. To rebalance the data, we can modify either the sampling or the update equations. We chose the first alternative since, in general, it led to faster convergence. If we take the example of $L_{O V R}$ then the sampling is modified as follows: draw $y=$ +1 with probability $\rho, y=-1$ with probability $1-\rho$. Then draw $x_{i}$ such that $y_{i}=y$.

\section{EXPERIMENTS}

In Section 5.1 we describe our experimental setup. In Section 5.2, we provide a detailed analysis of the different objective functions and parameters for three fine-grained subsets of ImageNet (Fungus, Ungulate, Vehicle). We believe that these fine-grained datasets can provide useful insights about the different objective functions since they correspond to different class densities as shown in [20]. In Section 5.4, we provide results on ImageNet10K.

\subsection{Experimental Setup}

In this section, we describe the datasets and image descriptors used in our experiments.

\begin{tabular}{|r|c|c|c|c|c|}
\hline & \multicolumn{2}{|c|}{ Total \# of } & \multicolumn{3}{c|}{ Partition } \\
\hline & images & classes & train & val & test \\
\hline Fungus & $88 \mathrm{~K}$ & 134 & $44 \mathrm{~K}$ & $5 \mathrm{~K}$ & $39 \mathrm{~K}$ \\
Ungulate & $183 \mathrm{~K}$ & 183 & $91.5 \mathrm{~K}$ & $5 \mathrm{~K}$ & $86.5 \mathrm{~K}$ \\
Vehicle262 & $226 \mathrm{~K}$ & 262 & $113 \mathrm{~K}$ & $5 \mathrm{~K}$ & $108 \mathrm{~K}$ \\
\hline ILSVRC10 & $1.4 \mathrm{M}$ & 1,000 & $1.2 \mathrm{M}$ & $50 \mathrm{~K}$ & $150 \mathrm{~K}$ \\
ImageNet10K & $9 \mathrm{M}$ & 10,184 & $4.5 \mathrm{M}$ & $50 \mathrm{~K}$ & $4.45 \mathrm{M}$ \\
\hline
\end{tabular}

TABLE 2

The datasets used in our evaluation.

Datasets. For our fine-grained image categorization experiments, we use three subsets of ImageNet: Fungus, Ungulate and Vehicle [20]. These datasets contain only the leaf nodes under the respective parent class (i.e. fungus, ungulate or vehicle) in the hierarchy of ImageNet. Unless stated otherwise, we used the following set-up: half of the data is used for training, $5 \mathrm{~K}$ images for validation and the remainder for testing.

For our large-scale experiments, we use ILSVRC 2010 [6] and ImageNet10K [20]. For ILSVRC2010, we follow the standard training/validation/testing protocol. For ImageNet10K, we follow [66] and use half of the data for training, 50K images for validation and the remainder for testing. The properties of the datasets are summarized in Table 2.

In all experiments, we compute the (flat) top- 1 or top- 5 accuracy per class and report the average as in [20], [66], [82]. We could also have used a hierarchical loss which takes into account the fact that some errors are more costly than others. The choice of a flat $v s$. a hierarchical loss is directly related to the choice of the cost function $\Delta$ (see section 3). In preliminary experiments, this choice had only very limited impact on the ranking of the different objective functions. This effect was also observed during the ILSVRC 2010 and 2011 competitions which might explain why the hierarchical measure was dropped in 2012. In what follows we report only accuracies with a flat loss.

Image Descriptors. Before feature extraction, we resize the 
images to $100 \mathrm{~K}$ pixels (if larger) while keeping the aspect ratio. We extract approx. 10K SIFT descriptors [50] at 5 scales for $24 \times 24$ patches every 4 pixels. The SIFT descriptors are reduced from 128-dim to 64-dim using PCA. They are then aggregated into an image-level signature (e.g. BOV or FV) using a probabilistic visual vocabulary, i.e. a Gaussian Mixture Model (GMM). By default, we use Fisher Vectors (FV) with $N=256$ Gaussians. We also report results with the bag of visual words (BOV) given its popularity in large-scale classification [20], [82]. Our default BOV uses $N=1,024$ codewords. Note that our purpose is not to compare the BOV and FV representations in this work (see [16] for such a comparison). Unless it is stated otherwise, we use spatial pyramids with $R=4$ regions (the entire image and three horizontal stripes).

Our experiments on large-scale datasets require image signatures to be compressed. For the FV we employ Product Quantization (PQ) [35]. We use sub-vectors of 8 dimensions and 8 bits per sub-vector. This setting was shown to yield minimal loss of accuracy in [66]. For the BOV we use Scalar Quantization with 8 bits per dimension as suggested in [20]. Signatures are decompressed on-the-fly by the SGD routines [66].

\subsection{Analysis on Fine Grained Datasets}

Distinguishing between semantically related categories which are also visually very similar is referred to as fine-grained visual categorization (see [23] for a study of the relationship between semantic and visual similarity in ImageNet). It has several applications such as the classification of mushrooms for edibility, animals for environmental monitoring or vehicles for traffic related applications. In this section we analyze the performance of the objective functions described in Section 3 in the context of fine-grained visual categorization. We report results on three subsets of ImageNet: Fungus, Ungulate and Vehicle [20].

\section{Comparison between SGD and Batch Methods.}

It is known that SGD can perform as well as batch solvers for OVR SVMs at a fraction of the cost [11], [67]. However, public SGD solvers do not exist for other SVM formulations such as w-OVR, MUL, RNK or WAR. Hence, as a sanity check, we compared our w-OVR and MUL SGD solvers to publicly available batch solvers. We compared our w-OVR SGD to LibSVM [15] and MUL SGD to LibLINEAR [26] and $\mathrm{SVM}^{\text {light }}$ [39]. Given the cost of training batch solvers, we restrict our experiments to the small dimensional BOV vectors ( $N=1,024 \times R=4 \rightarrow 4,096$ dimensions). Furthermore, we perform training on subsets of the full training sets: for each class we use 10, 25, 50 and 100 random samples within the training set. We repeat the experiments 5 different times for 5 random subsets of training images. Top- 1 classification accuracy is reported in Figure 1 and 2.

These experiments show that stochastic and batch solvers perform on par for w-OVR and MUL SVMs. Somewhat surprisingly, SGD solvers even seem to have a slight edge over batch solvers, especially for the MUL SVM. This might be because SGD uses as stopping criterion the accuracy on the (a) w-OVR SVM: LibSVM vs. SGD

\begin{tabular}{|r|c|c|c|}
\hline & \multicolumn{3}{|c|}{ LibSVM (batch) / SGD (online) } \\
\hline & Fungus & Ungulate & Vehicle \\
\hline 10 & $12 / 7$ & $31 / 18$ & $107 / 39$ \\
25 & $95 / 16$ & $175 / 36$ & $835 / 119$ \\
50 & $441 / 38$ & $909 / 67$ & $3,223 / 271$ \\
100 & $1,346 / 71$ & $3,677 / 133$ & $11,679 / 314$ \\
\hline
\end{tabular}

(b) MUL SVM: SVM ${ }^{l i g h t} v s$. SGD

\begin{tabular}{|r|c|c|c|}
\hline & \multicolumn{3}{|c|}{ SVM $^{\text {light }}$ (batch) / SGD (online) } \\
\hline & Fungus & Ungulate & Vehicle \\
\hline 10 & $45 / 36$ & $324 / 81$ & $557 / 209$ \\
25 & $99 / 72$ & $441 / 198$ & $723 / 369$ \\
50 & $198 / 261$ & $855 / 420$ & $1,265 / 747$ \\
100 & $972 / 522$ & $1,674 / 765$ & $3,752 / 1,503$ \\
\hline
\end{tabular}

\section{TABLE 3}

Average training time (in CPU seconds) on Fungus, Ungulate, Vehicle for 10, 25, 50, 100 training samples per class using 4,096-dim BOV.

validation set measured with the true target loss (top-1 loss in this case). In contrast, batch solvers stop upon convergence of a surrogate objective function on the training set. The training times of SGD and batch solvers are reported in Table 3 (in CPU seconds on 32GBs RAM double quad-core multithreaded servers using a single CPU). As expected, the CPU time of SGD solvers is significantly smaller than for batch solvers and the difference increases for larger training sets.

\section{Importance of Data Rebalancing in OVR.}

In this section we investigate the effect of data rebalancing for OVR SVMs. Note that more sophisticated strategies could also be used, as in [70]. Here we simply emphasize that in terms of performance, it is always beneficial to crossvalidate the imbalance parameter of one-vs-rest classifiers. We compare unweighted OVR (u-OVR) and weighted OVR (wOVR). Since we do the reweighting by biasing the sampling, we introduce the imbalance parameter $\beta=(1-\rho) / \rho$ which is the (average) number of negatives sampled for each positive. $\beta=1$ corresponds to the natural rebalancing of the data (giving the same weight to positives and negatives). The solid lines in Figure 3 show the results with w-OVR and the dashed lines with u-OVR. We ran experiments with BOV descriptors with $N=1024$ Gaussians and FV descriptors with $N=8,16$, 64, and 256 Gaussians using a pyramid with $R=4$ regions.

We can draw the following conclusions. First, rebalancing makes a significant difference for smaller dimensional features, but has less impact on high-dimensional features. Second, it is crucial to correctly set the parameter $\beta$, as natural rebalancing generally does not lead to the best results. Indeed, the optimal $\beta$ depends on many factors including the dataset, the feature type and the feature dimensionality. We observe empirically that the optimal $\beta$ typically increases with the number of classes. For instance, on ILSVRC 2010 and ImageNet10K respectively, the best w-OVR results are obtained with $\beta=64$ and $\beta=256$ (with the $4 \mathrm{~K}$-dim BOV features). In the following, we will use the weighted version of OVR, i.e. w-OVR.

\section{Explicit vs. Implicit Regularization.}




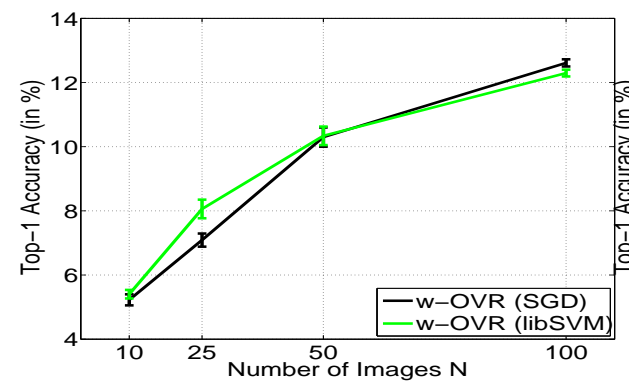

(a) Fungus

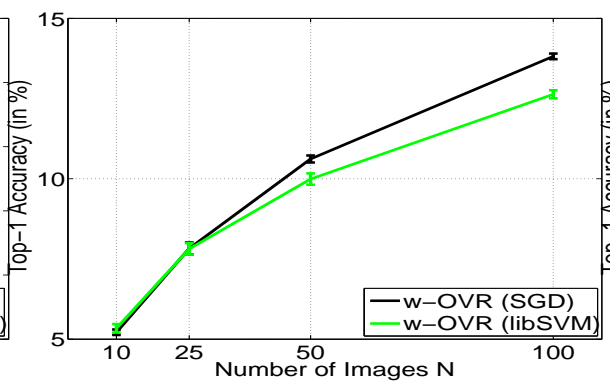

(b) Ungulate

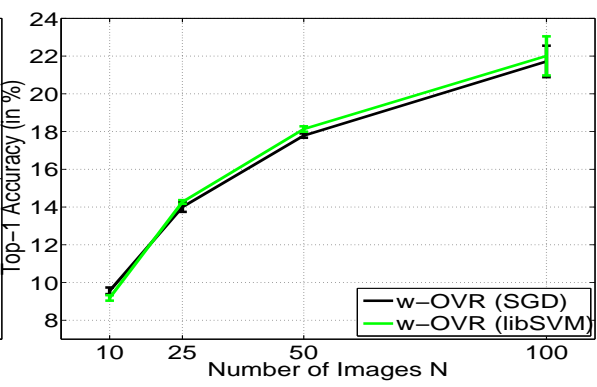

(c) Vehicle

Fig. 1. Comparison of SGD and libSVM for optimizing the w-OVR SVM using 4,096-dim BOV descriptors. From left to right: Fungus, Ungulate and Vehicle.

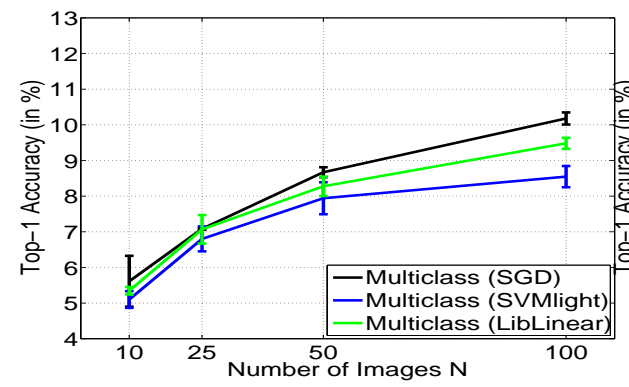

(a) Fungus

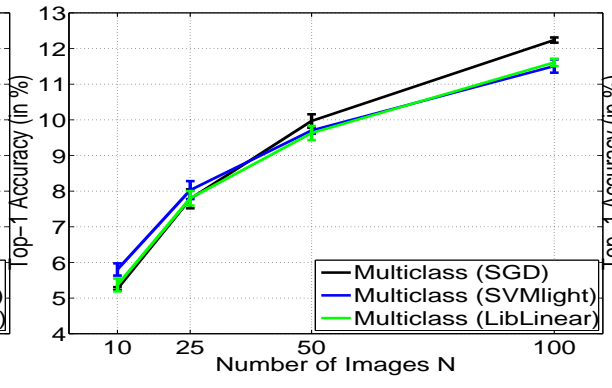

(b) Ungulate

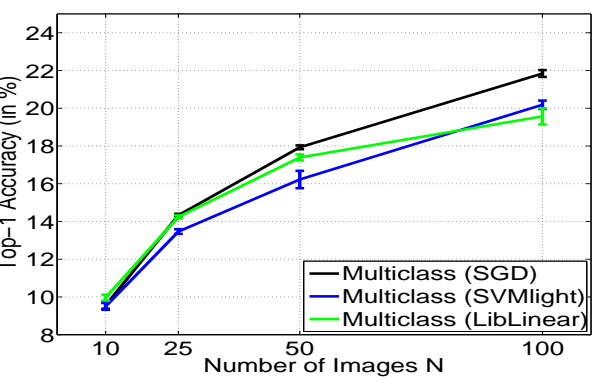

(c) Vehicle

Fig. 2. Comparison of SGD, SVM ${ }^{\text {light }}$ and LibLinear for optimizing the MUL SVM using using 4,096-dim BOV. From left to right: Fungus, Ungulate and Vehicle.

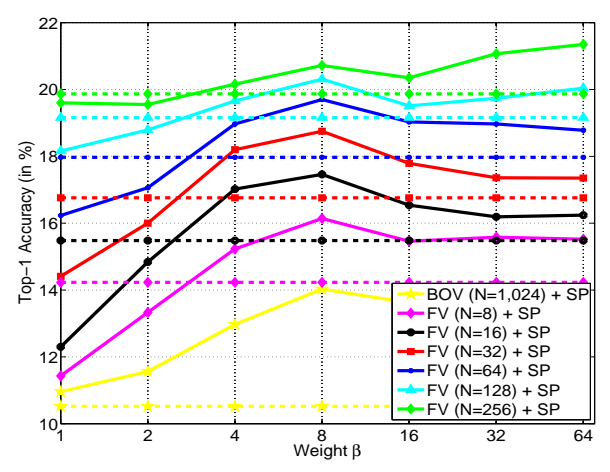

(a) Fungus

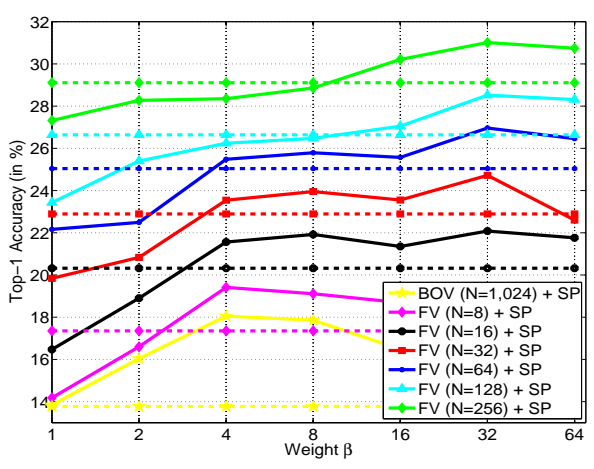

(b) Ungulate

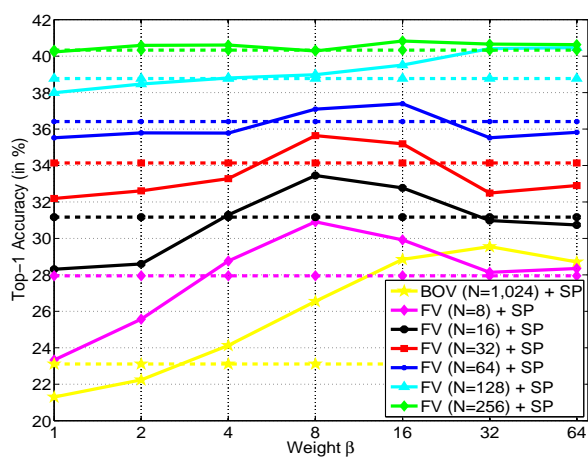

(c) Vehicle

Fig. 3. Influence of data rebalancing in weighted One-vs-Rest (w-OVR). The value of the weight parameter indicates the number of negative training samples for one positive sample (solid lines). The results of unweighted One-vs-Rest (u-OVR) are shown with dashed lines.

We run experiments on high-dimensional features (the 130K-dim FV for $N=256$ and $R=4$ ), i.e. for which regularization is supposed to have a significant impact. The following experiments focus on w-OVR but we obtained very similar results for MUL, RNK and WAR. We compare three regularization strategies:

(i) Using explicit regularization $(\lambda>0)$ and a decreasing step size $\eta_{t}=1 /(\lambda(t+t 0)$ [10]. Setting $t_{0}$ correctly is of paramount importance for fast convergence and Bottou [10] proposes a heuristic to set $t_{0}$ automatically ${ }^{1}$. We attempted to cross-validate $t_{0}$, but always found the optimal value to be very close to the one predicted by Bottou's heuristic.

(ii) Using explicit regularization $\lambda>0$ and a fixed step size $\eta_{t}=\eta$.

(iii) Using no regularization $(\lambda=0)$ and a fixed step size $\eta_{t}=\eta$, i.e. implicitly regularizing with the number of iterations.

Results are provided in Figure 4 with $\beta$ set by cross-

1. This heuristic is based on the assumption that the input vectors are $\ell_{2}$ normalized and uses the fact that the norm of the optimal $w$, denoted $w^{*}$, is bounded [67]: $\left\|w^{*}\right\| \leq 1 / \sqrt{\lambda} . t_{0}$ is set so that the norm of $w$ during the first iterations is comparable to this bound. 


\begin{tabular}{|l|c|c|c|}
\hline & Fungus & Ungulate & Vehicle \\
\hline no replacement & $\mathbf{1 9 . 7 5}$ & $\mathbf{2 7 . 8 6}$ & 38.40 \\
\hline replacement & 19.55 & 27.79 & $\mathbf{3 9 . 5 3}$ \\
\hline
\end{tabular}

TABLE 4

Comparison of sampling with and without replacement for w-OVR. Top-1 accuracy (\%) on Fungus, Ungulate, Vehicle using 130K-dim FVs.

validation. All three strategies perform similarly showing that implicit regularization with fixed step size can be an effective learning strategy. We observe that for smaller datasets it is important to stop early for optimal performance. This is consistent with regularization being less important when training data is plentiful. We also experimented with the implicit regularization with early stopping on the small-scale PASCAL VOC 2007 dataset [25] and observed a small drop of performance compared to explicit regularization (from $62.1 \%$ to $60.2 \%$ ) which might indicate that this strategy is better suited to large-scale datasets.

\section{Analysis of Sampling in w-OVR.}

In this section, we show the effect of sampling the negative data points with or without replacement during SGD training for w-OVR. We experiment with our best features, i.e. the 130K-dim FV for compatibility with our previous results. The results are reported in Table 4 in terms of top-1 accuracy. We can see that sampling without replacement does not improve the accuracy significantly. In what follows, we draw negative samples with replacement.

\section{Influence of Training Data Size.}

We now compare the four different objective functions: wOVR, MUL, RNK and WAR. We are interested in understanding whether the amount of training data has an impact on their relative performance. We run experiments with $10,25,50$ and 100 training samples per class. The experiments are repeated 100 times for 10 and 25 training samples and 5 times for 50 and 100 training samples. We use the 4,096 dimensional BOV descriptors and report the results in Figure 5.

Despite its simplicity and its supposed sub-optimality, wOVR provides a competitive performance. It seems that RNK has an edge over the three other objective functions for a very small number of training samples. We provide in Table 5 the numerical results for 10 and 25 training samples. To understand whether the observed differences are significant, we performed two types of tests. We performed the sign test [68], [65], which counts the number of times an algorithm performs better than the other one, and the paired t-test, which tests whether the observed differences in accuracies are purely due to random errors. We show in Table 6 the probability for both tests to reject the null hypothesis, i.e. the probability that the observed differences happen by chance. In all cases the probabilities are close to zero, i.e. the null hypothesis is rejected with high confidence, thus indicating that the modest improvement of RNK with respect to w-OVR, MUL and WAR for a small number of training samples is significant.

Our interpretation of this result is as follows. The OVR objective function for a given class can be written as a sum of losses over samples $\left(x_{i}, y_{i}\right)$. In contrast, using the weighted (a) 10 training images per class

\begin{tabular}{|l|c|c|c|}
\hline & \multicolumn{3}{|c|}{ Mean $(\mu) /$ Std deviation $(\sigma)$} \\
\hline & Fungus & Ungulate & Vehicle \\
\hline W-OVR & $5.26 / 0.20$ & $5.24 / 0.14$ & $9.66 / 0.20$ \\
MUL & $5.06 / 0.28$ & $5.11 / 0.16$ & $9.43 / 0.19$ \\
RNK & $\mathbf{5 . 6 1} / \mathbf{0 . 6 4}$ & $\mathbf{5 . 5 2} / \mathbf{0 . 2 6}$ & $\mathbf{1 0 . 1 8} / \mathbf{0 . 3 3}$ \\
WAR & $5.26 / 0.25$ & $5.19 / 0.17$ & $9.52 / 0.25$ \\
\hline
\end{tabular}

(b) 25 training images per class

\begin{tabular}{|l|c|c|c|}
\hline & \multicolumn{3}{|c|}{ Mean $(\mu) /$ Std deviation $(\sigma)$} \\
\hline & Fungus & Ungulate & Vehicle \\
\hline W-OVR & $7.52 / 0.25$ & $7.77 / 0.15$ & $14.05 / 0.19$ \\
MUL & $6.90 / 0.19$ & $7.57 / 0.19$ & $13.75 / 0.23$ \\
RNK & $\mathbf{7 . 8 9} / \mathbf{0 . 2 4}$ & $\mathbf{8 . 1 1} / \mathbf{0 . 1 8}$ & $\mathbf{1 4 . 6 5} / \mathbf{0 . 3 2}$ \\
WAR & $7.66 / 0.24$ & $7.80 / 0.18$ & $13.83 / 0.27$ \\
\hline
\end{tabular}

TABLE 5

The average and standard deviation of the top-1 accuracy in on Fungus, Ungulate, Vehicle with 10 and 25 training images per class (repeated 100 times) using 4,096-dim BOV.

ranking framework of Usunier et al. [75] (see also our section 3.2) the MUL, RNK and WAR objective functions can be written as sums of losses over triplets $\left(x_{i}, y_{i}, y\right)$. Since the number of triplets is $C$ times larger than the number of samples, one could expect the latter objective functions to have an edge over OVR in the small sample regime. However, while the RNK objective function is an unweighted sum over triplets, the MUL and WAR objective functions correspond to weighted sums that give more importance to the top of the list. In other words, because of the weighting, the effective number of triplets is much smaller than the actual number of triplets in the objective function. For instance, in the case of MUL, only the top triplet has a non-zero weight which means that the effective number of triplets equals the number of samples. This may explain why eventually MUL does not have an edge over OVR in the small sample regime. Similarly, the WAR weighting can be viewed as a smooth interpolation between the MUL weighting and the RNK weighting. Although the WAR weighting - which is inversely proportional to the triplet rank - makes sense for our classification goal, it is by no means guaranteed to be optimal. While Usunier et al. propose alternative weight profiles (see section 6 in [75]), we believe that such weights should be learned from the data in order to give full strength to the WAR objective function. However, it is not straightforward to devise a method to learn these weights from the data. Furthermore, from our experiments, the optimal weighting profile seems to be dependent on the number of training samples.

\section{Influence of Class Density.}

We observed in Fig 5 that MUL can perform poorly, especially on the very challenging Fungus dataset. Note that this is the densest of the three fine-grained subsets ${ }^{2}$.

To better understand the influence of data density, we first experimented on synthetic data. The data is composed of 2D

2. The density of a dataset [20] is defined as the mean distance between all pairs of categories, where the distance between two categories in a hierarchy is the height of their lowest common ancestor. Small values imply dense datasets and indicate a more challenging recognition problem. 


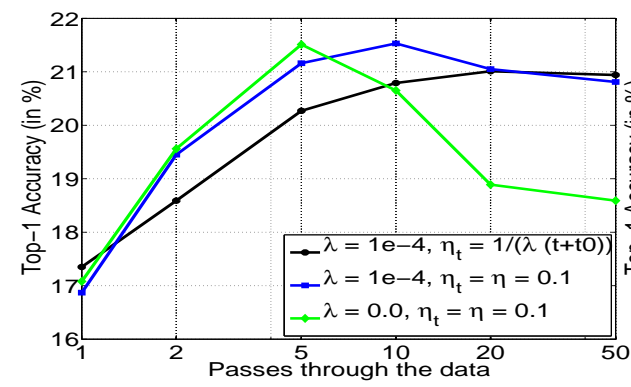

(a) Fungus134

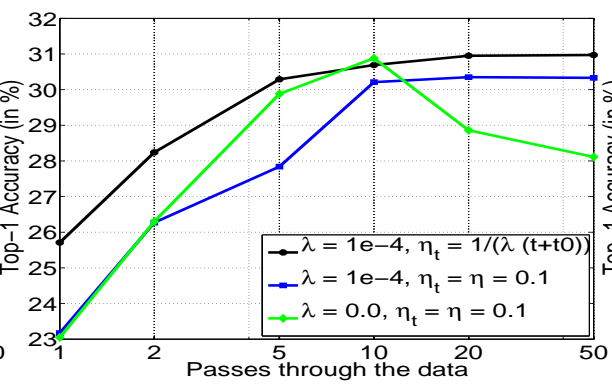

(b) Ungulate183

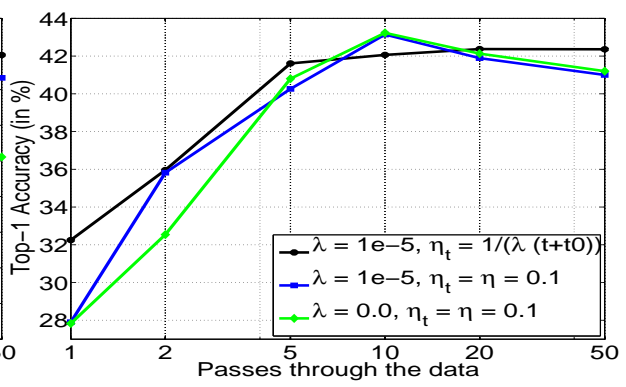

(c) Vehicle262

Fig. 4. Impact of regularization on w-OVR. Results on Fungus, Ungulate, Vehicle using 130K-dim FVs. One pass here means seeing all positives for a given class + (on average) $\beta$ times more negatives.

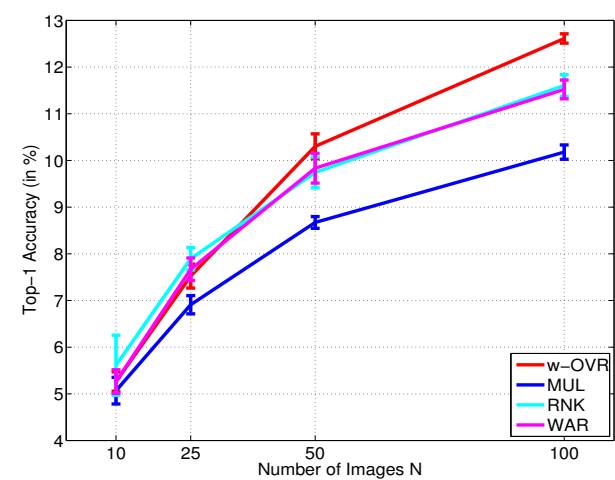

(a) Fungus

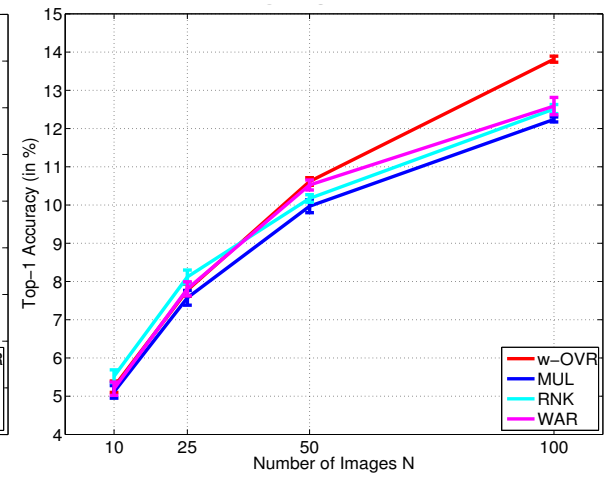

(b) Ungulate

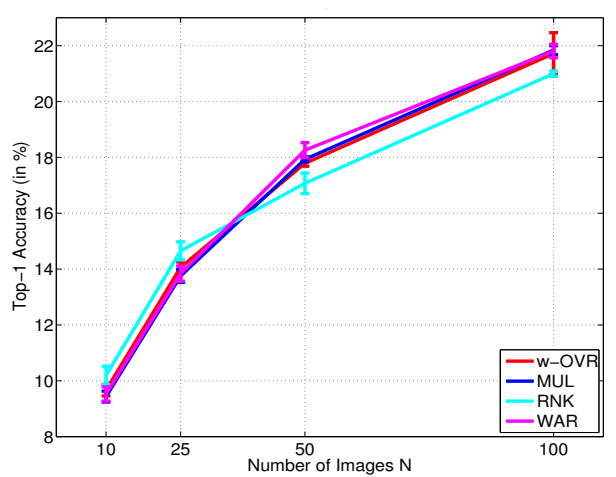

(c) Vehicle

Fig. 5. Comparison of four objective functions ( $w-O V R, M U L, R N K$ and WAR) as a function of the number of training images on Fungus, Ungulate, Vehicle using 4,096-dim BOV

\begin{tabular}{|c|c|c|c|}
\hline & \multicolumn{3}{|c|}{ p-values (t-test, sign test) } \\
\hline & RNK vs MUL & RNK vs WAR & RNK vs w-OVR \\
\hline F10 & $9.82 \mathrm{e}-12,5.07 \mathrm{e}-20$ & $5.48 \mathrm{e}-6,5.52 \mathrm{e}-17$ & $4.81 \mathrm{e}-7,9.44 \mathrm{e}-20$ \\
F25 & $9.32 \mathrm{e}-51,3.15 \mathrm{e}-28$ & $1.39 \mathrm{e}-10,2.49 \mathrm{e}-08$ & $3.55 \mathrm{e}-20,1.42 \mathrm{e}-13$ \\
\hline U10 & $3.16 \mathrm{e}-34,5.10 \mathrm{e}-25$ & $5.97 \mathrm{e}-25,3.31 \mathrm{e}-18$ & $1.03 \mathrm{e}-20,3.77 \mathrm{e}-21$ \\
U25 & $2.69 \mathrm{e}-36,1.23 \mathrm{e}-23$ & $1.84 \mathrm{e}-18,1.04 \mathrm{e}-16$ & $4.49 \mathrm{e}-26,3.31 \mathrm{e}-18$ \\
\hline V10 & $1.36 \mathrm{e}-35,5.58 \mathrm{e}-19$ & $2.06 \mathrm{e}-26,5.58 \mathrm{e}-19$ & $1.23 \mathrm{e}-26,3.31 \mathrm{e}-18$ \\
V25 & $8.34 \mathrm{e}-35,3.31 \mathrm{e}-18$ & $2.74 \mathrm{e}-30,3.31 \mathrm{e}-18$ & $3.02 \mathrm{e}-33,3.31 \mathrm{e}-18$ \\
\hline
\end{tabular}

TABLE 6

RNK method in comparison with all of the other SGD methods (F:Fungus, U:Ungulate, V:Vehicle). 10 and 25 refer to the number of training samples per class.

Features: 4,096-dim BOV.

\begin{tabular}{|l|c|c|c|c|c|c|}
\hline$\sigma=$ & 0.1 & 0.25 & 1.0 & 2.0 & 3.0 & 5.0 \\
\hline \hline w-OVR & 100.00 & 96.30 & 47.04 & 33.08 & 27.78 & 24.48 \\
\hline MUL & 100.00 & 95.70 & 44.96 & 26.70 & 23.32 & 22.36 \\
\hline RNK & 100.00 & 96.28 & 47.76 & 33.24 & 27.66 & 24.30 \\
\hline WAR & 100.00 & 96.32 & 47.48 & 32.98 & 27.62 & 24.62 \\
\hline
\end{tabular}

TABLE 7

Comparison of the accuracies (\%) between w-OVR, MUL, RNK and WAR on synthetic data. $\sigma$ is the standard deviation of the Gaussians.

points drawn from 5 different Gaussian distributions with fixed means and variable standard deviations $(0.10 .25,1,2,3$ and 5), see Fig 6 for a standard deviation of 0.25 . As the standard deviation increases, the overlap between classes increases

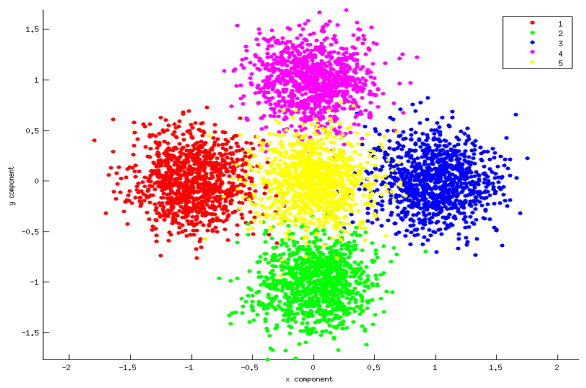

Fig. 6. Synthetic dataset with standard deviation 0.25 .

significantly. We can see from Table 7 that all classifiers yield perfect results when the data are well separable, but that MUL performs worse than all the other methods in case of overlapping distributions.

We also experimented on the Fungus dataset. To vary the density, we sub-sampled different numbers of classes: 10, 25, 50,100 . A small number of classes would typically result in a lower class density. We show results with BOV vectors in Figure 7. The ordering of the classifiers does not seem to be dependent on the number of classes and therefore on the data density. We conducted similar experiments on Ungulate and Vehicle and obtained comparable results.

From the results of our synthetic and real data experiments, it is therefore difficult to argue for the merits of one classifier 


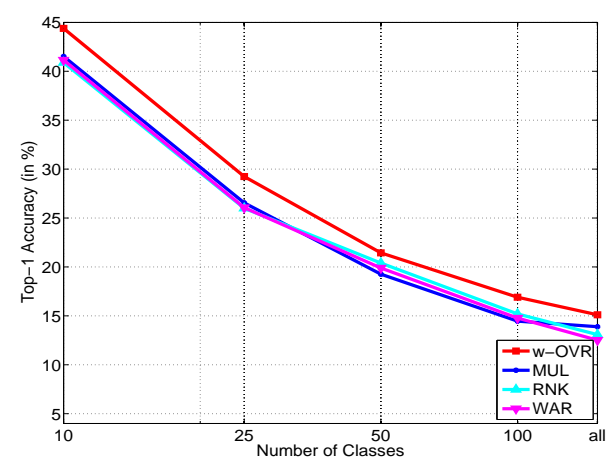

Fig. 7. Top-1 accuracy as a function of the number of classes.

or the other for denser or sparser datasets.

\section{Influence of Feature Dimensionality.}

Again, we compare the four objective functions (w-OVR, MUL, RNK and WAR), but this time we investigate whether the feature dimensionality has an impact on the relative performance. Fig. 8 shows results for FVs of different dimensionality, i.e., the number of Gaussians varies from $N=8$ to 256. We observe again that the w-OVR provides a competitive performance. We also observe that, as the feature dimensionality increases, the difference between the different objective functions becomes smaller (this is also observed on the ILSVRC 2010 dataset, see [55]).

Hence, for high-dimensional features, the learning objective function seems to have little impact. Put into the perspective of the statistical learning theory [3], this implies that the impact of the different choices of surrogate loss is mitigated as the capacity of the classifier increases. In [3], the $\psi(\cdot)$ function defines how the performance in the surrogate loss transfers to the target loss (see Theorem 10 in [3]). For each surrogate loss there is a corresponding $\psi(\cdot)$ function. In our experiments the different learning objectives corresponding to different surrogate losses lead to similar, yet, good results. Therefore, in these experiments, the capacity of the classifiers at hand is "large" enough, relative to the difficulty of the dataset, so large that the difference of the corresponding $\psi(\cdot)$ functions is almost canceled.

\subsection{Large-Scale Experiments on ILSVRC2010}

We report our results using the ILSVRC 2010 subset of ImageNet that contains $1 \mathrm{~K}$ classes.

Results. We provide a comparison of the OVR, MUL, RNK and WAR SVMs. We do not report detailed results as on the fine-grained datasets, as conclusions are very similar, see [55] for detailed experiments on ILSVRC 2010. We report results for the 4K-dim BOVs and 130K-dim FVs, see table 8. We observe that the conclusions drawn from the smaller finegrained datasets are still valid. First, rebalancing the positives and negatives is important in the case of OVR for lowdimensional training samples. Second, w-OVR is a competitive strategy despite its theoretical sub-optimality compared to the ranking objective functions. Third, for high-dimensional FV

\begin{tabular}{|c|l|c|c|c|c|c|}
\hline & & u-OVR & w-OVR & MUL & RNK & WAR \\
\hline \hline \multirow{2}{*}{ Top-1 } & BOV & 15.8 & 26.4 & 22.7 & 20.8 & 24.1 \\
& FV & 45.9 & 45.7 & 46.2 & 46.1 & 46.1 \\
\hline \hline \multirow{2}{*}{ Top-5 } & BOV & 28.8 & 46.4 & 38.4 & 41.2 & 44.2 \\
& FV & 63.7 & 65.9 & 64.8 & 65.8 & 66.5 \\
\hline
\end{tabular}

TABLE 8

Comparison of u-OVR, w-OVR, MUL, RNK and WAR. Accuracy (in \%) on ILSVRC 2010 for 4K-dim BOV and 130K-dim FV.

\begin{tabular}{|c|c|c|c|c|c|}
\hline & u-OVR & w-OVR & MUL & RNK & WAR \\
\hline \hline BOV 4K-dim & 3.8 & 7.5 & 6.0 & 4.4 & 7.0 \\
\hline FV 130K-dim & - & 19.1 & - & - & 17.9 \\
\hline
\end{tabular}

TABLE 9

Top-1 accuracy (in \%) on ImageNet10K.

features all methods perform similarly. The difference between the best and worst performing methods is $0.5 \%$ at top- 1 and $2.8 \%$ at top-5.

Comparison with the State-of-the-Art. In what follows, we report results for the top-5 accuracy which is the standard measure used during the competition. We note that the two winning teams at ILSVRC 2010 reported better results than our $66.5 \%$ at top-5. [66] reports $74.3 \%$ by combining SIFT and color descriptors and by using 520K-dim FVs while [48] reports $71.8 \%$ by combining SIFT and LBP descriptors as well as multiple encoding techniques and spatial pyramids. While better features can indeed increase accuracy, this is out of the scope of our paper. We note however that our $66.5 \%$ is slightly above the $65 \%$ reported by the challenge winners using their single best feature [6].

\subsection{Large-Scale Experiments on ImageNet10K}

We now report our results using on ImageNet10K which contains approx. 10K classes and 9M images.

Results. In Table 9, we report results for the 4K-dim BOV and the 130K-dim FV using top-1 accuracy as in [20], [66]. Given the cost of running the experiments on the high-dimensional FVs, we carried-out experiments only with the two objective functions which performed best on ILSVRC 2010: w-OVR and WAR. As a conclusion, w-OVR performs better than more complex objective functions (at least on this dataset with those features). Some example classes from ImageNet10K which reflect the difficulty of the dataset along with the top- 1 perclass accuracies are presented in Figure 9. Figure 10 gives more detailed per-class results.

Comparison with the State-of-the-Art. We now compare our results with results at the scale of $O\left(10^{4}\right)$ categories [20], [66], [82]. Compared to [20], our BOV results are on par (even slightly better since we report $7.5 \%$ while they report $6.4 \%$ ) and our FV results are significantly (almost 3 times) better due to the use of higher-dimensional features. However, our focus in this work is not on features but on (linear) classifier learning strategies. 


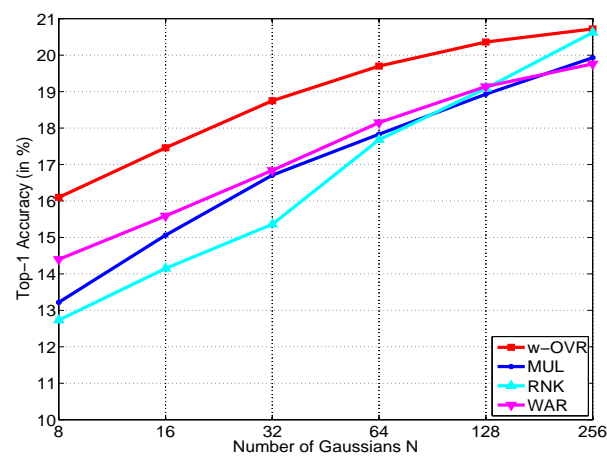

(a) Fungus

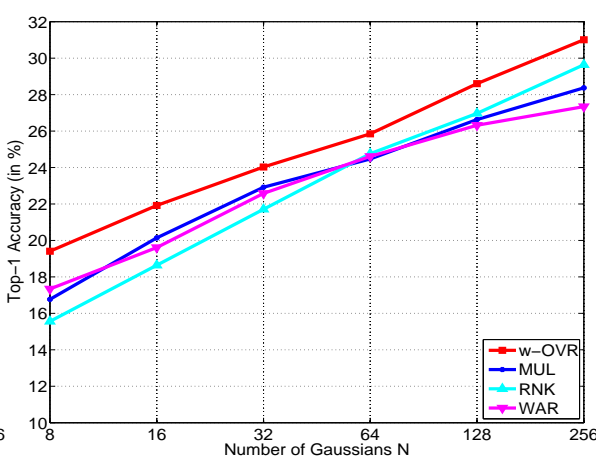

(b) Ungulate

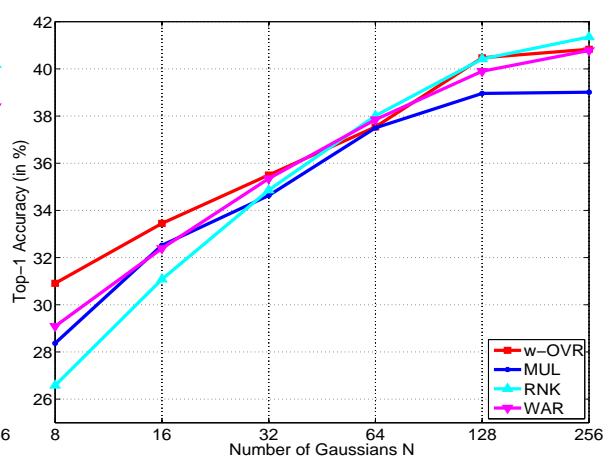

(c) Vehicle

Fig. 8. Comparison of Top-1 Accuracy between the w-OVR, MUL, RNK and WAR SVMs as a function of the number of Gaussians used to compute the FV (i.e. as a function of the FV dimensionality). No spatial pyramids were used to speed-up these experiments.

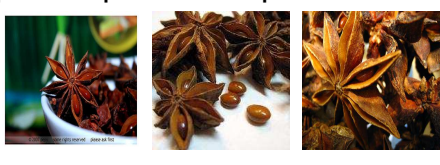

(a) Star Anise (92\%)

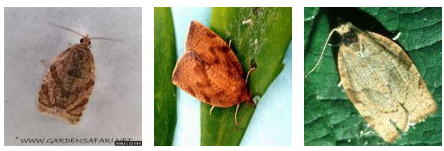

(e) Tortrix $(25 \%)$

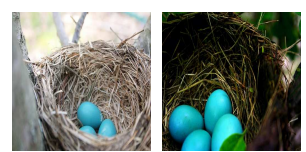

(b) Nest Egg (87\%)

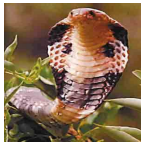

(f) Egyptian cobra (10\%)
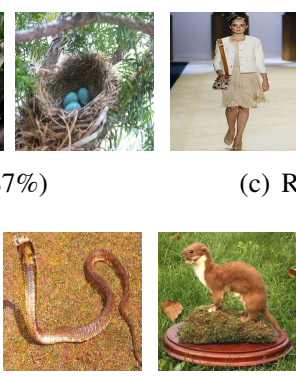

(g) Weasel $(5 \%)$

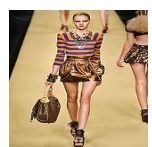

(c) Ready to Wear (76\%)
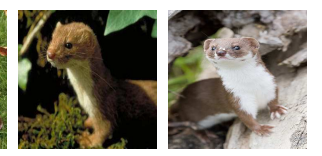

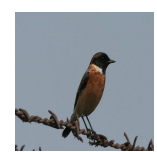

(d) Stonechat $(50 \%)$
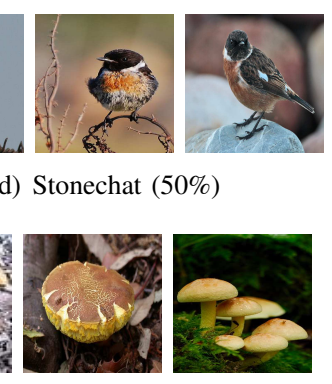

(h) Felt fungus $(0 \%)$

Fig. 9. ImageNet10K results (top-1 accuracy in \%) obtained with w-OVR and 130K-dim Fisher vectors. (a-d) Sample classes from the best performing ones until the ones with accuracy 50\%. (e-h) Sample classes with performance around $25 \%$ until the worst performing ones.

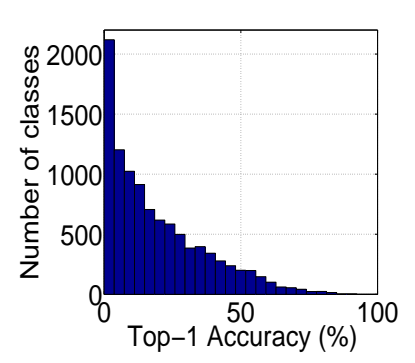

(a)

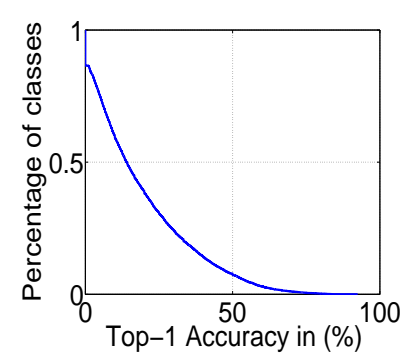

(b)

Fig. 10. Top-1 accuracies on ImageNet10K. Left: histogram of the top- 1 accuracies of all the $10 \mathrm{~K}$ classes. Right: percentage of classes whose top- 1 accuracy is above a threshold.

Weston et al., who used a different ImageNet subset in their experiments, show that WAR outperforms OVR [82] on BOV descriptors, where their OVR baseline did not do any reweighting of the positives/negatives, i.e. it is similar to our u-OVR. We also observed that WAR significantly outperforms u-OVR. However, we show that w-OVR performs significantly better than u-OVR and slightly better than WAR.

As for [66], Sánchez and Perronnin use w-OVR with the natural rebalancing $(\beta=1)$. We show that selecting $\beta$ by cross-validation can have a significant impact on accuracy: using the same features, we improve their baseline by an

absolute $2.4 \%$, from $16.7 \%$ to $19.1 \%$. It is interesting to note that while rebalancing the data has little impact on the $130 \mathrm{~K}$ dim FV on ILSVRC 2010, it has a significant impact on ImageNet10K. This is not in contradiction with our previous statement that different objective functions perform similarly on high-dimensional features. We believe this is because there is no such thing as "high-dimensional" features. Features are only high-dimensional with respect to the complexity of the problem and especially the number of classes. While 130Kdim is high-dimensional with respect to the $1 \mathrm{~K}$ categories of ILSVRC 2010, it is not high-dimensional anymore with respect to the $10 \mathrm{~K}$ categories of ImageNet10K.

Le et al. [42] and Krizhevsky et al. [40] also report results on the same subset of $10 \mathrm{~K}$ classes. They report respectively a top-1 per-image accuracy of $19.2 \%$ and $32.6 \%{ }^{3}$. Our perimage accuracy is $21.0 \%$. However, we did not optimize for this metric which might lead to better results. Note that our study is not about comparing FVs to features learned with deep architectures. We could have used the learned features of [42], [40] in our study if they had been available, i.e., we could use as features the output of any of the intermediate

3. While it is standard practice to report per-class accuracy on this dataset (see [20], [66]), [42], [40] report a per-image accuracy. This results in a more optimistic number since those classes which are over-represented in the test data also have more training samples and therefore have (on average) a higher accuracy than those classes which are under-represented. This was clarified through a personal correspondence with the first authors of [42], [40]. 
layers.

Timing for ImageNet10K for 130K-dim FVs. For the computation we used a small cluster of machines with $16 \mathrm{CPUs}$ and 32GB of RAM. The feature extraction step (including SIFT description and FV computation) took approx. $250 \mathrm{CPU}$ days, the learning of the w-OVR SVM approx. $400 \mathrm{CPU}$ days and the learning of the WAR SVM approx. 500 CPU days. Note that w-OVR performs slightly better than WAR and is much easier to parallelize since the classifiers can be learned independently.

\section{Conclusion}

In this work, we have studied visual classification on a largescale, i.e. when we have to deal with a large number of classes, a large number of images and high dimensional features. Two main conclusions have emerged from our work. The first one is that, despite its theoretical suboptimality, one-vsrest is a very competitive training strategy to learn SVMs. Furthermore, one-vs-rest SVMs are easy to implement and to parallelize, e.g. by training the different classifiers on multiple machines/cores. However, to obtain state-of-the-art results, properly cross-validating the imbalance between positive and negative samples is a must. The second major conclusion is that stochastic training is very well suited to our largescale setting. Moreover simple strategies such as implicit regularization with early stopping and fixed-step-size updates work well in practice. Following these good practices, we were able to improve the state-of-the-art on a large subset of $10 \mathrm{~K}$ classes and 9M images from $16.7 \%$ Top-1 accuracy to $19.1 \%$.

Acknowledgements. The INRIA LEAR team acknowledges financial support from the QUAERO project supported by OSEO, the French State agency for innovation, the European integrated project AXES, the ERC grant ALLEGRO and the GARGANTUA project funded by the Mastodons program of CNRS. The authors wish to warmly thank Matthijs Douze and Mattis Paulin for providing a public implementation of the online learning code described in this article.

\section{REFERENCES}

[1] E. L. Allwein, R. E. Schapire, and Y. Singer. Reducing multiclass to binary: A unifying approach for margin classifiers. In ICML, 2000. 3

[2] B. Bai, J. Weston, D. Grangier, R. Collobert, O. Chapelle, and K. Weinberger. Supervised semantic indexing. In CIKM, 2009. 6

[3] P. L. Bartlett, M. I. Jordan, and J. D. McAuliffe. Convexity, classification, and risk bounds. In NIPS, 2003. 11

[4] S. Bengio, J. Weston, and D. Grangier. Label embedding trees for large multi-class tasks. In NIPS, 2010. 1, 3

[5] Y. Bengio, A. Courville, and P. Vincent. Representation learning: a review and new perspectives. 3

[6] A. Berg, J. Deng, and L. Fei-Fei. ILSVRC 2010. http://www.imagenet.org/challenges/LSVRC/2010/index. 1, 6, 11

[7] A. Bergamo, L. Torresani, and A. Fitzgibbon. PICODES: Learning a compact code for novel-category recognition. In NIPS, 2011. 3

[8] A. Beygelzimer, V. Dani, T. P. Hayes, J. Langford, and B. Zadrozny. Error limiting reductions between classification tasks. In ICML, 2005. 3

[9] A. Bordes, L. Bottou, P. Gallinari, and J. Weston. Solving multiclass support vector machines with LaRank. In ICML, 2007. 1

[10] L. Bottou. SGD. http://leon.bottou.org/projects/sgd. 4, 5, 8

[11] L. Bottou and O. Bousquet. The tradeoffs of large scale learning. In NIPS, 2007. 1, 5, 7
[12] Y.-L. Bourreau, F. Bach, Y. LeCun, and J. Ponce. Learning mid-level features for recognition. In CVPR, 2010. 2

[13] G. Burghouts and J.-M. Geusebroek. Performance evaluation of local colour invariants. CVIU, 2009. 2

[14] P. K. Chan and S. J. Stolfo. On the accuracy of meta-learning for scalable data mining. JIIS, 1997. 3

[15] C.-C. Chang and C.-J. Lin. LIBSVM: A library for support vector machines. ACM TIST, 2011. Software available at http://www.csie.ntu. edu.tw/ cjlin/libsvm. 4, 7

[16] K. Chatfield, V. Lempitsky, A. Vedaldi, and A. Zisserman. The devil is in the details: an evaluation of recent feature encoding methods. In BMVC, 2011. 3, 7

[17] K. Crammer and Y. Singer. On the algorithmic implementation of multiclass kernel-based vector machines. JMLR, 2002. 1, 3, 4

[18] G. Csurka, C. Dance, L. Fan, J. Willamowski, and C. Bray. Visual categorization with bags of keypoints. In ECCV SLCV workshop, 2004 2

[19] J. Dean, G. Corrado, R. Monga, M. Devin, Q. Le, M. Mao, M. Ranzato, A. Senior, P. Tucker, K. Yang, and A. Ng. Large scale distributed deep networks. In NIPS, 2012. 3

[20] J. Deng, A. Berg, K. Li, and L. Fei-Fei. What does classifying more than 10,000 image categories tell us? In $E C C V, 2010.1,3,6,7,9,11$, 12

[21] J. Deng, W. Dong, R. Socher, L.-J. Li, K. Li, and L. Fei-Fei. Imagenet: A large-scale hierarchical image database. In CVPR, 2009. 1, 2

[22] J. Deng, S. Satheesh, A. Berg, and L. Fei-Fei. Fast and balanced: efficient label tree learning for large scale object recognition. In NIPS, 2011. 1

[23] T. Deselaers and V. Ferrari. Visual and semantic similarity in imagenet. In CVPR, 2011. 7

[24] T. G. Dietterich and G. Bakiri. Solving multiclass learning problems via error-correcting output codes. JAIR, 1995. 3

[25] M. Everingham, L. V. Gool, C. Williams, J. Winn, and A. Zisserman. The pascal visual object classes (VOC) challenge. IJCV, 2010. 2, 9

[26] R.-E. Fan, K.-W. Chang, C.-J. Hsieh, X.-R. Wang, and C.-J. Lin. LIBLINEAR: A library for large linear classification. JMLR, 2008. 7

[27] J. Farquhar, S. Szedmak, H. Meng, and J. Shawe-Taylor. Improving bag-of-keypoints image categorisation. Technical report, University of Southampton, 2005. 2

[28] V. Franc and S. Sonnenburg. Optimized cutting plane algorithm for support vector machines. In ICML, 2008. 4

[29] T. Gao and D. Koller. Discriminative learning of relaxed hierarchy for large-scale visual recognition. In ICCV, 2011. 3

[30] J. Gehrke, R. Ramakrishnan, and V. Ganti. Rainforest - a framework for fast decision tree construction of large datasets. DMKD, 2000. 3

[31] Y. Gong and S. Lazebnik. Comparing data-dependent and dataindependent embeddings for classification and ranking of internet images. In $C V P R, 2011.2$

[32] D. Grangier, F. Monay, and S. Bengio. A discriminative approach for the retrieval of images from text queries. In $E C M L, 2006.3$

[33] C.-J. Hsieh, K.-W. Chang, C.-J. Lin, S. S. Keerthi, and S. Sundararajan. A dual coordinate descent method for large-scale linear svm. In ICML, 2008. 4

[34] K. Jarrett, K. Kavukcuoglu, M. Ranzato, and Y. LeCun. What is the best multi-stage architecture for object recognition? In ICCV, 2009. 3

[35] H. Jégou, M. Douze, and C. Schmid. Product quantization for nearest neighbor search. IEEE TPAMI, 2011. 3, 7

[36] H. Jégou, F. Perronnin, M. Douze, J. Sánchez, P. Pérez, and C. Schmid. Aggregating local image descriptors into compact codes. IEEE TPAMI, accepted. 3

[37] T. Joachims. Making large-scale support vector machine learning practical. In Advances in kernel methods. 1999. 4

[38] T. Joachims. Optimizing search engines using clickthrough data. In ACM SIGKDD, pages 133-142. ACM, 2002. 1, 3, 4

[39] T. Joachims. Training linear svms in linear time. In ACM SIGKDD, 2006. 7

[40] A. Krizhevsky, I. Sutskever, and G. Hinton. Image classification with deep convolutional neural networks. In NIPS, 2012. 2, 3, 12

[41] S. Lazebnik, C. Schmid, and J. Ponce. Beyond bags of features: Spatial pyramid matching for recognizing natural scene categories. In $C V P R$, 2006. 2

[42] Q. Le, M. Ranzato, R. Monga, M. Devin, K. Chen, G. Corrado, J. Dean, and $\mathrm{A}$. Ng. Building high-level features using large scale unsupervised learning. In ICML, 2012. 2, 3, 12

[43] Y. LeCun, B. Boser, J. Denker, D. Henderson, R. Howard, W. Hubbard, and L. Jackel. Handwritten digit recognition with a back-propagation network. In NIPS, 1989. 3

[44] Y. LeCun, L. Bottou, G. Orr, and K. Muller. Efficient backprop. In Neural Networks: Tricks of the trade. Springer, 1998. 1

[45] Y. LeCun, F. Huang, and L. Bottou. Learning methods for generic object 
recognition with invariance to pose and lighting. In $C V P R, 2004.3$

[46] T. Lee, Y. Lin, and G. Wahba. Multicategory support vector machines: Theory and application to the classification of microarray data and satellite radiance data. JASA, 2004. 3

[47] L. Li, H. Su, E. Xing, and L. Fei-Fei. Object bank: A high-level image representation for scene classification and semantic feature sparsification. In NIPS, 2010. 3

[48] Y. Lin, F. Lv, S. Zhu, M. Yang, T. Cour, K. Yu, L. Cao, and T. Huang. Large-scale image classification: Fast feature extraction and SVM training. In $C V P R, 2011.1,2,3,5,11$

[49] Y. Lin, F. Lv, S. Zhu, K. Yu, M. Yang, and T. Cour. Large-scale image classification: fast feature extraction and svm training. In CVPR, 2011. 3

[50] D. G. Lowe. Distinctive image features from scale-invariant keypoints. IJCV, 2004. 2, 7

[51] S. Maji and A. Berg. Max-margin additive classifiers for detection. In ICCV, 2009. 2

[52] M. Marszalek and C. Schmid. Constructing category hierarchies for visual recognition. In $E C C V, 2008.3$

[53] M. Mehta, R. Agrawal, and J. Rissanen. Sliq: A fast scalable classifier for data mining. In $E D B T, 1996.3$

[54] S. Nowozin and C. Lampert. Structured Learning and Prediction in Computer Vision. Foundations and Trends in Computer Graphics and Vision, 2011. 1

[55] F. Perronnin, Z. Akata, Z. Harchaoui, and C. Schmid. Towards good practice in large-scale learning for image classification. In $C V P R, 2012$. 2, 11

[56] F. Perronnin and C. Dance. Fisher kernels on visual vocabularies for image categorization. In $C V P R, 2007.2$

[57] F. Perronnin, J. Sánchez, and Y. Liu. Large-scale image categorization with explicit data embedding. In $C V P R, 2010.2,3,5$

[58] F. Perronnin, J. Sánchez, and T. Mensink. Improving the Fisher kernel for large-scale image classification. In ECCV, 2010. 1, 5

[59] J. C. Platt. Fast training of support vector machines using sequential minimal optimization. In Advances in kernel methods. 1999. 3

[60] M. Rastegari, C. Fang, and L. Torresani. Scalable object-class retrieval with approximate and top-k ranking. In $I C C V, 2011.3$

[61] R. Rifkin and A. Klautau. In defense of one-vs-all classification. JMLR, 2004. 3

[62] M. Rohrbach, M. Stark, and B.Schiele. Evaluating knowledge transfer and zero-shot learning in a large-scale setting. In $C V P R, 2011.1,3,5$

[63] L. Rokach and O. Maimon. Top-down induction of decision trees classifiers - a survey. IEEE TSMC, 2005. 3

[64] B. C. Russell, A. Torralba, K. P. Murphy, and W. T. Freeman. Labelme: A database and web-based tool for image annotation. IJCV, 2008. 2

[65] S. L. Salzberg. On comparing classifiers: Pitfalls toavoid and a recommended approach. DMKD. 9

[66] J. Sánchez and F. Perronnin. High-dimensional signature compression for large-scale image classification. In $C V P R, 2011.1,3,5,6,7,11$, 12

[67] S. Shalev-Shwartz, Y. Singer, and N. Srebro. Pegasos: Primal estimate sub-gradient solver for SVM. In ICML, 2007. 1, 4, 5, 7, 8

[68] D. J. Sheskin. Handbook of Parametric and Nonparametric Statistical Procedures. Chapman \& Hall/CRC, 4 edition, 2007. 9

[69] J. Sivic and A. Zisserman. Video google: A text retrieval approach to object matching in videos. In ICCV, 2003. 2

[70] D. Tao, X. Tang, X. Li, and X. Wu. Asymmetric bagging and random subspace for support vector machines-based relevance feedback in image retrieval. IEEE Trans. Pattern Anal. Mach. Intell., 28(7), July 2006. 7

[71] A. Tewari and P. L. Bartlett. On the Consistency of Multiclass Classification Methods. JMLR, 2007. 3, 4

[72] A. Torralba, R. Fergus, and W. Freeman. 80 million tiny images: a large dataset for non-parametric object and scene recognition. IEEE TPAMI, 2008. 1, 2, 3

[73] L. Torresani, M. Szummer, and A. Fitzgibbon. Efficient object category recognition using classemes. In ECCV, 2010. 3

[74] I. Tsochantaridis, T. Joachims, T. Hofmann, and Y. Altun. Large margin methods for structured and interdependent output variables. JMLR, 2005. 4

[75] N. Usunier, D. Buffoni, and P. Gallinari. Ranking with ordered weighted pairwise classification. In $I C M L, 2009.1,3,4,5,9$

[76] J. C. van Gemert, C. J. Veenman, A. W. M. Smeulders, and J. M. Geusebroek. Visual word ambiguity. IEEE TPAMI, 2010.

[77] A. Vedaldi and A. Zisserman. Efficient additive kernels via explicit feature maps. In CVPR, 2010. 2

[78] A. Vedaldi and A. Zisserman. Sparse kernel approximations for efficient classification and detection. In $C V P R, 2012.3$

[79] V. Vural and J. G. Dy. A hierarchical method for multi-class support vector machines. In ICML, 2004. 3

[80] G. Wang, D. Hoiem, and D. Forsyth. Learning image similarity from
Flickr groups using stochastic intersection kernel machines. In ICCV, 2009. 1,3

[81] J. Wang, J. Yang, K. Yu, F. Lv, T. Huang, and Y. Gong. Localityconstrained linear coding for image classification. In CVPR, 2010. 2

[82] J. Weston, S. Bengio, and N. Usunier. Large scale image annotation: Learning to rank with joint word-image embeddings. ECML, 2010. 1, $3,5,6,7,11,12$

[83] J. Weston, S. Bengio, and N. Usunier. Wsabie: Scaling up to large vocabulary image annotation. In IJCAI, 2011. 3

[84] J. Weston and C. Watkins. Multi-class support vector machines Technical report, Department of Computer Science, Royal Holloway, University of London, 1998. 4

[85] J. Weston and C. Watkins. Support vector machines for multi-class pattern recognition. In ESANN, 1999. 3

[86] J. Xu, T. Liu, M. Lu, H. Li, and W. Ma. Directly optimizing evaluation measures in learning to rank. In SIGIR, 2008. 1, 5

[87] J. Yang, K. Yu, Y. Gong, and T. S. Huang. Linear spatial pyramid matching using sparse coding for image classification. In CVPR, 2009.

[88] Y. Yue, T. Finley, F. Radlinski, and T. Joachims. A support vector method for optimizing average precision. In SIGIR, 2007. 1, 5

[89] B. Zhao, L. Fei-Fei, and E. Xing. Large-scale category structure aware image categorization. In NIPS, 2011. 1

[90] Z. Zhou, K. Yu, T. Zhang, and T. Huang. Image classification using super-vector coding of local image descriptors. In ECCV, 2010. 3

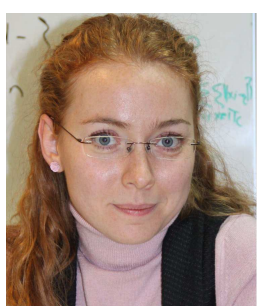

Zeynep Akata received her MSc degree in 2010 from the Media Informatics department of RWTH Aachen University, Germany. She is currently working on her PhD in the CV team of the Xerox Research Centre Europe and the LEAR team at INRIA Grenoble. She is working on image classification for large scale datasets and is interested in learning methods for classification. She is a student member of IEEE.

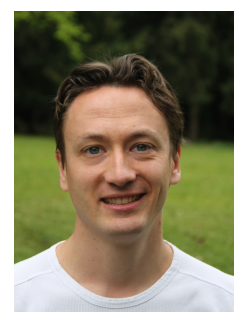

Florent Perronnin holds an Engineering degree from the Ecole Nationale Supérieure des Télécommunications and a Ph.D. degree from the Ecole Polytechnique Fédérale de Lausanne. In 2005, he joined the Xerox Research Centre Europe in Grenoble where he currently manages the CV team. His main interests are in the application of machine learning to computer vision tasks such as image classification, retrieval or segmentation.

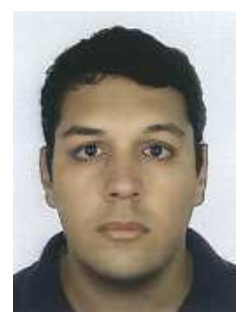

Zaid Harchaoui graduated from the Ecole $\mathrm{Na}$ tionale Superieure des Mines, France, in 2004, and obtained his Ph.D. degree from ParisTech, Paris, France. Since 2010, he is a permanent researcher in the LEAR team, INRIA Grenoble, France. His research interests include statistical machine learning, kernel-based methods, signal processing, and computer vision.

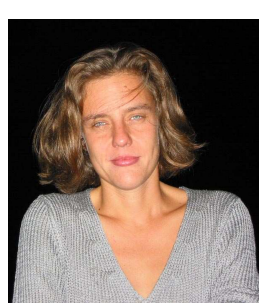

Cordelia Schmid holds a M.S. degree in computer science from the University of Karlsruhe and a doctorate from the Institut National Polytechnique de Grenoble. She is a research director at INRIA Grenoble where she directs the LEAR team. In 2006, she was awarded the Longuet-Higgins prize for fundamental contributions in computer vision that have withstood the test of time. In 2012, she obtained an ERC advanced grant for "Active large-scale learning for visual recognition". She is a fellow of IEEE. 